\title{
The Cosmic Microwave Background Temperature Bispectrum from Scalar Perturbations Induced by Primordial Magnetic Fields
}

\author{
Chiara Caprini, 1 , 田 Fabio Finelli, $2,3,4$, 团 Daniela Paoletti, ${ }^{5,2}$, 团 and Antonio Riotto ${ }^{6,7}$, 鸟 \\ ${ }^{1}$ CEA, IPhT \& CNRS, URA 2306, F-91191 Gif-sur-Yvette, France \\ ${ }^{2}$ INAF-IASF Bologna, via Gobetti 101, I-40129 Bologna, Italy \\ ${ }^{3}$ INAF-OAB, Osservatorio Astronomico di Bologna, via Ranzani 1, I-40127 Bologna, Italy \\ ${ }^{4}$ INFN, Sezione di Bologna, Via Irnerio 46, I-40126 Bologna, Italy \\ ${ }^{5}$ Dip. di Fisica, Università degli studi di Ferrara and INFN, via Saragat 1, I-44100 Ferrara, Italy \\ ${ }^{6}$ CERN, PH-TH Division, CH-1211 Geneva 23, Switzerland \\ ${ }^{7}$ INFN, Sezione di Padova, via Marzolo 8, Padova I-35131, Italy
}

(Dated: 1st November 2018)

\begin{abstract}
We evaluate the angular bispectrum of the CMB temperature anisotropy at large angular scale due to a stochastic background of primordial magnetic fields. The shape of non-Gaussianity depends on the spectral index of the magnetic field power spectrum and is peaked in the squeezed configuration for a scale-invariant magnetic spectrum. By using the large angular part of the bispectrum generated by magnetic fields, the present bounds on non-Gaussianity set a limit on the amplitude of the primordial magnetic field of the order of $\mathcal{O}(10)$ nGauss for the scale-invariant case and $\mathcal{O}(20)$ nGauss for the other spectral indexes.
\end{abstract}

\section{INTRODUCTION}

Cosmological inflation [1] has become the dominant paradigm to understand the initial conditions for the Cosmic Microwave Background (CMB) anisotropies and structure formation. This picture has recently received further spectacular confirmation by the Wilkinson Microwave Anisotropy Probe (WMAP) five year set of data [2]. Present [2] and future [3] experiments may be sensitive to the non-linearities of the cosmological perturbations at the level of second- or higher-order perturbation theory. The detection of these non-linearities through the non-Gaussianity $(\mathrm{NG})$ in the CMB [4] has become one of the primary experimental targets.

A possible source of NG could be primordial in origin, being specific to a particular mechanism for the generation of the cosmological perturbations. This is what makes a positive detection of NG so relevant: it might help in discriminating among competing scenarios which otherwise might be indistinguishable. Indeed, various models of inflation, firmly rooted in modern particle physics theory, predict a significant amount of primordial NG generated either during or immediately after inflation when the comoving curvature perturbation becomes constant on superhorizon scales [4]. While single-field [5] and two(multi)-field [6] models of inflation generically predict a tiny level of NG, 'curvaton-type models', in which a significant contribution to the curvature perturbation is generated after the end of slow-roll inflation by the perturbation in a field which has a negligible effect on inflation, may predict a high level of NG 7, 8]. Alternatives to the curvaton model are those models characterised by the curvature perturbation being generated by an inhomogeneity in the decay rate [9, 10] or the mass [11] or of the particles responsible for the reheating after inflation. Other opportunities for generating the curvature perturbation occur at the end of inflation [12] and during preheating [13]. All these models generate a level of NG which is local as the NG part of the primordial curvature perturbation is a local function of the Gaussian part, being generated on superhorizon scales. In momentum space, the three point function, or bispectrum, arising from the local NG is dominated by the so-called "squeezed" configuration, where one of the momenta is much smaller than the other two and it is parametrized by the non-linearity parameter $f_{\mathrm{NL}}^{\text {loc }}$. Other models, such as DBI inflation [14] and ghost inflation [15], predict a different kind of primordial NG, called "equilateral", because the three-point function for this kind of NG is peaked on equilateral configurations, in which the lengths of the three wave-vectors forming a triangle in Fourier space are equal [16]. The equilateral NG is parametrized by an amplitude $f_{\mathrm{NL}}^{\text {equil }}$ [17]. Present limits on NG are summarised by $-9<f_{\mathrm{NL}}^{\text {loc }}<111$ and $-151<f_{\mathrm{NL}}^{\text {equil }}<253$ at $95 \% \mathrm{CL}[2,18]$.

\footnotetext{
*Electronic address: chiara.caprini@cea.fr

${ }^{\dagger}$ Electronic address: finelli@iasfbo.inaf.it

‡Electronic address: paoletti@iasfbo.inaf.it

$\S$ Electronic address: antonio.riotto@cern.ch
} 
On the other hand there might exist other sources of primordial NG in the CMB anisotropies beyond the primordial ones related to the dynamics of inflation. One interesting possibility is the contribution to the non-Gaussian signal in the CMB anisotropies from a stochastic background of primordial magnetic fields. Large scale magnetic fields are almost everywhere in the universe, from galaxies up to those present in galaxy clusters and in the inter-cluster medium [19]. The dynamo effect provides a mechanism to explain the observed magnetic fields associated to galaxies, whereas those associated to clusters may be generated by gravitational compression. Both these mechanisms require an initial magnetic seed, although with different amplitude and different correlation length.

Possible explanations for this initial seed have driven the interest in primordial magnetic fields generated in the early universe. A stochastic background of primordial magnetic fields (PMFs) generated in the early universe with a mean amplitude well below micro-Gauss level can leave imprints on the temperature and polarisation anisotropy pattern of the cosmic microwave background (CMB). The impact of a stochastic background of PMFs onto CMB anisotropies has distinctive imprints, such as a contribution in temperature which is larger than the CMB angular power spectrum sourced by scalar cosmological perturbation at high $\ell$ and a contribution in polarisation which include either $B B$ (generated by vector and tensor perturbations or by Faraday rotation [20]) or parity-odd correlators as $T B$ (generated by an helical component [21]).

As we mentioned, another distinctive imprint of PMF in CMB anisotropies is its non-Gaussian nature. The CMB signature of this type which has been first considered in the literature is due to a homogeneous PMF. A homogeneous magnetic field with fixed direction breaks spatial isotropy in the universe, and therefore leads to non-zero correlations between multipoles at different $\ell,\left\langle a_{\ell-1, m} a_{\ell+1, m}\right\rangle \neq 0$ [22]. This effect has been first proposed in [23], and arises through the generation of vector metric pertubations from the Alfvén waves magnetically induced in the primordial fluid. Recently, it has been reanalysed and found to reproduce, for a sufficiently high magnetic field amplitude, some of the anomalies of the CMB large scale fluctuations observed by WMAP such as the north-south asymmetry and the quadrupole-octopole alignment [24].

This effect is related to the presence of a homogeneous magnetic field (or equivalently, a stochastic magnetic field with correlation length larger than the horizon today). On the other hand, the CMB contribution of a stochastic background of PMFs, modelled as a fully inhomogeneous component, is intrinsically non-Gaussian: the PMFs energymomentum tensor, the Lorentz force acting on baryons are quadratic in the magnetic field $\mathbf{B}(\eta, \mathbf{x})$, which is randomly distributed with a Gaussian distribution function. The source terms to the Einstein-Boltzmann system are therefore $\chi$-distributed, leading to a PMF contribution to CMB fully non-Gaussian. Higher order statistical moments of the energy-momentum tensor of PMFs are therefore non-vanishing at leading order and are calculable with minimal assumptions, such as cutting sharply the power spectrum beyond a certain scale $k_{D}$ [25].

Non-gaussianities from PMFs are much less studied than those generated in inflationary methods. The study of the three point statistics of the PMF energy-momentum tensor in 25] is limited to the simplest particular collinear configuration. Nevertheless, due to the presence of a contribution from the collinear configuration, one deduces that non-gaussianities from PMFs can be different from the inflationary case, in which the collinear contribution is generically negligible with respect to the equilateral and squeezed ones (see however [26]). In this paper we focus on the three point statistics of the PMF energy density, studying the contribution of all three configurations. By using the large scale relation between temperature anisotropies and PMF energy density given in Ref. [27], we compute the temperature bispectrum and compare its contribution to the non-Gaussian statistics in the CMB anisotropies with the present observational bounds. We also compare our results with those of the very recent paper [28].

Our paper is organised as follows. In section II we introduce the stochastic background of primordial magnetic fields and discuss the infrared behaviour of the spectra of its energy-density. In section III and IV we discuss the CMB temperature spectrum and bispectrum induced by PMF on large scales. Section V is devoted to the analytic computation of the magnetic energy density bispectrum $\left\langle\rho_{B}(\mathbf{k}) \rho_{B}(\mathbf{q}) \rho_{B}(\mathbf{p})\right\rangle$ in general and for the collinear, squeezed and equilateral configurations. In Section VI we insert these results into the CMB temperature bispectrum on large scales, and we give an estimation of the signal in section VII. In the first Appendix we derive analytic approximations to some integrals of Bessel functions which are useful to calculate both the spectrum and the bispectrum, and in the second Appendix we give the details for the exact computation of the energy density bispectrum in the collinear case for $n=2,-2$.

\section{PRIMORDIAL STOCHASTIC MAGNETIC FIELD}

We adopt notations consistent with [29, 30]:

$$
B_{i}(\mathbf{x})=\int \frac{d^{3} k}{(2 \pi)^{3}} e^{-i \mathbf{k} \cdot \mathbf{x}} B_{i}(\mathbf{k}) \rightarrow \delta(\mathbf{k})=\int \frac{d^{3} x}{(2 \pi)^{3}} e^{i \mathbf{k} \cdot \mathbf{x}},
$$


where the definition of the delta function comes from $\int d^{3} k /(2 \pi)^{3} e^{-i \mathbf{k} \cdot \mathbf{x}} \delta(\mathbf{k})=1 /(2 \pi)^{3}$. With these conventions, the magnetic field power spectrum (defined as the Fourier transform of the two point correlation function) is ${ }^{1}$ :

$$
\begin{aligned}
\left\langle B_{i}(\mathbf{k}) B_{j}^{*}(\mathbf{q})\right\rangle & =(2 \pi)^{3} \delta^{3}(\mathbf{k}-\mathbf{q})\left(\delta_{i j}-\hat{k}_{i} \hat{k}_{j}\right) P_{B}(k) \\
P_{B}(k) & =A k^{n}, \quad k \leq k_{D},
\end{aligned}
$$

where $\hat{k}_{i}=k_{i} / k, A$ is a normalisation constant, $n$ the spectral index and $k_{D}$ the upper cutoff. Using the above equations we can define the mean square of the magnetic field as

$$
\left\langle B^{2}(\mathbf{x})\right\rangle=\frac{A}{\pi^{2}} \frac{k_{D}^{n+3}}{n+3} .
$$

If we are interested in the mean amplitude of the magnetic field on a given characteristic scale, we smooth the power spectrum over the chosen scale using a Gaussian filter: we have then $\left.\left\langle B^{2}(\mathbf{x})\right\rangle\right|_{\lambda}=B_{\lambda}^{2}$ with

$$
B_{\lambda}^{2}=\frac{1}{\pi^{2}} \int d k k^{2} P_{B}(k) e^{-k^{2} \lambda^{2}}=\frac{A}{2 \pi^{2}} \frac{\Gamma[(n+3) / 2]}{\lambda^{n+3}},
$$

so that

$$
B_{\lambda}^{2}=\frac{\left\langle B^{2}\right\rangle}{2} \frac{n+3}{\left(k_{D} \lambda\right)^{n+3}} \Gamma\left(\frac{n+3}{2}\right) .
$$

We also define the adimensional quantity $\Omega_{B}^{\text {tot }}$ given by the ratio of the magnetic and the total radiation energy densities:

$$
\Omega_{B}^{\text {tot }}=\frac{\left\langle B^{2}\right\rangle}{8 \pi \rho_{\text {rel }}} \simeq 10^{-7} \frac{\left\langle B^{2}(\mathbf{x})\right\rangle}{\left(10^{-9} \text { Gauss }\right)^{2}},
$$

where for the last equality we have used $\rho_{\text {rel }}\left(\eta_{0}\right) \simeq 2 \times 10^{-51} \mathrm{GeV}^{4}$, and $\eta_{0}$ denotes the conformal time today.

The upper cutoff $k_{D}$ corresponds to the damping scale, representing the dissipation of magnetic energy due to the generation of magneto-hydrodynamic waves [31, 32]. Alfvén waves are the most effective in dissipating magnetic energy, and in [32] it is demonstrated that around recombination the damping occurs at scales $k^{-1} \lesssim k_{D}^{-1} \simeq V_{A} L_{\text {Silk }}$, where $V_{A}$ is the Alfvén speed and $L_{\text {Silk }}$ the Silk damping scale at recombination. Strictly speaking, Alfvén waves are oscillatory perturbations superimposed on a homogeneous magnetic component, and the Alfvén speed depends on the amplitude of the homogeneous component. In the cosmological context where the magnetic field is purely stochastic, the amplitude of this component can be taken as the one of a 'low frequency' component obtained by smoothing the magnetic field amplitude over a sufficiently large scale [33]. This scale corresponds to the Alfvén scale at recombination, $k_{A}^{-1} \simeq V_{A} \eta_{\text {rec }}$ : magnetic modes on lager scales, in fact, do not have time to oscillate before recombination [32]. One has therefore $k_{D} / k_{A}=\eta_{\mathrm{rec}} / L_{\mathrm{Silk}} \simeq 10$, and $V_{A}^{2}=B_{L_{A}}^{2} /(4 \pi(\rho+p))$. Consequently, the upper cutoff at the epoch of recombination is given by

$$
k_{D} \simeq \frac{1}{L_{\mathrm{Silk}}} \sqrt{\frac{16 \pi}{3} \frac{\rho_{\mathrm{rel}}}{B_{L_{A}}^{2}}}=\frac{1}{L_{\mathrm{Silk}}} \frac{1}{\sqrt{\Omega_{B}^{\mathrm{tot}}}} \sqrt{\frac{(2 \pi)^{n+3}}{(n+3) \Gamma((n+3) / 2)}}\left(\frac{k_{A}}{k_{D}}\right)^{\frac{n+3}{2}} .
$$

Finiteness of the total magnetic energy density implies $n>-3$. In the rest of the paper we keep the spectral index as a free parameter, when possible; however, in order to carry on our calculations analytically we sometimes need to specify it. For example, in Section $\square$, we choose the values $n=2$ and $n=-2 . n=2$ is the lowest possible value for a magnetic field generated by a causal process [34], such as a phase transition [35], or a charge separation process operating around recombination [36]. A magnetic field generated during inflation [37] (or by any other a-causal process such as, for example, in pre big bang theories [38]), can take any value of $n>-3$. However, because of Nucleosynthesis constraints [39], only for very red spectra the magnetic field can assume sufficiently high amplitudes to have an impact in the CMB. Therefore, in the following we choose the value $n=-2$ (for some analytic calculations), or $n \rightarrow-3$ when possible.

\footnotetext{
${ }^{1}$ In this paper we neglect the possible presence of an helical component for the magnetic field, see for example 21]
} 
The spatial part of the energy momentum tensor of the magnetic field is

$$
\tau_{i j}(\mathbf{x})=\frac{1}{4 \pi}\left[\frac{1}{2} \delta_{i j} B_{l}(\mathbf{x}) B_{l}(\mathbf{x})-B_{i}(\mathbf{x}) B_{j}(\mathbf{x})\right],
$$

and the magnetic energy density

$$
\begin{aligned}
& \rho_{B}(\mathbf{x})=\tau_{i i}(\mathbf{x})=\frac{1}{8 \pi} B^{2}(\mathbf{x}) \\
& \rho_{B}(\mathbf{k})=\frac{1}{8 \pi} \int \frac{d^{3} p}{(2 \pi)^{3}} B_{i}(\mathbf{k}-\mathbf{p}) B_{i}(\mathbf{p}) .
\end{aligned}
$$

As we will see in the next section, to calculate the CMB temperature spectrum from the Sachs Wolfe effect we need the power spectrum of the magnetic energy density:

$$
\left\langle\rho_{B}(\mathbf{k}) \rho_{B}^{*}(\mathbf{q})\right\rangle \equiv(2 \pi)^{3} \delta(\mathbf{k}-\mathbf{q})\left|\rho_{B}(k)\right|^{2}=\frac{2}{(8 \pi)^{2}} \delta(\mathbf{k}-\mathbf{q}) \int d^{3} p P_{B}(\mathbf{p}) P_{B}(|\mathbf{k}-\mathbf{p}|)\left(1+\mu^{2}\right),
$$

the second equality is obtained using Eq. (10), and $\mu=\hat{p} \cdot \widehat{\mathbf{k}-\mathbf{p}}$. Therefore

$$
\left|\rho_{B}(k)\right|^{2}=\frac{1}{256 \pi^{5}} \int d^{3} p P_{B}(\mathbf{p}) P_{B}(|\mathbf{k}-\mathbf{p}|)\left(1+\mu^{2}\right) \text {. }
$$

As demonstrated in Ref. [27], $\left|\rho_{B}(k)\right|^{2}$ goes to zero at a wavenumber corresponding to twice the magnetic field spectrum cutoff, $k=2 k_{D}$. Eq. (21) of [27] gives the behaviour of $\left|\rho_{B}(k)\right|^{2}$ at large scales $k \ll k_{D}$ and for spectral indexes $n>-3 / 2$ : the generic behaviour in this case is white noise [27]

$$
\left|\rho_{B}(k)\right|^{2} \simeq \frac{A^{2} k_{D}^{2 n+3}}{32 \pi^{4}(2 n+3)} .
$$

For $n=-2$, an exact calculation as in Refs. [27, 40] gives the behaviour for $\left|\rho_{B}(k)\right|^{2}$ at large scales $k \ll k_{D}$ as

$$
\left|\rho_{B}(k)\right|^{2} \simeq \frac{3 A^{2}}{512 \pi^{2} k}
$$

For $n<-3 / 2$ we use the approximated formula given by Ref. [29]:

$$
\left|\rho_{B}(k)\right|^{2} \simeq \frac{3 A^{2}}{128 \pi^{4}} \frac{n}{(2 n+3)(n+3)} k^{2 n+3} .
$$

For $n=-2$, expressions (14) and (15) are in agreement concerning the dependence on the wavenumber, but the numerical factor differs by a factor $\pi^{2} / 8$.

\section{CMB TEMPERATURE SPECTRUM AT LARGE ANGULAR SCALES}

We use the characterisation of the CMB temperature anisotropy induced by a stochastic background of primordial magnetic fields given in [40]. It is useful to define the adimensional magnetic energy parameter in $k$-space

$$
\Omega_{B}(\mathbf{k})=\frac{\rho_{B}(\mathbf{k})}{\rho_{\text {rel }}}
$$

From the initial conditions given in [27, 40] we parametrize the temperature anisotropy as

$$
\frac{1}{4} \delta_{\gamma}+\psi=\frac{\alpha}{4} \Omega_{B}(\mathbf{k})
$$

where $\alpha \sim 0.1$ is a multiplication constant required since the above equation would be exact with $\alpha=1$ in the radiation era. Therefore, the temperature anisotropy is given in terms of this quantity as

$$
\frac{\Theta_{\ell}^{(0)}\left(\eta_{0}, \mathbf{k}\right)}{2 \ell+1}=\frac{\alpha}{4} \Omega_{B}(\mathbf{k}) j_{\ell}\left(k\left(\eta_{0}-\eta_{\mathrm{dec}}\right)\right),
$$


where $j_{\ell}$ is the spherical Bessel function and $\eta_{0}, \eta_{\mathrm{dec}}$ denote conformal time respectively today and at decoupling. The CMB power spectrum is therefore [30]:

$$
C_{\ell}^{B}=\frac{2}{\pi} \int_{0}^{\infty} d k k^{2} \frac{\left\langle\Theta_{\ell}^{(0)}\left(\eta_{0}, \mathbf{k}\right) \Theta_{\ell}^{(0) *}\left(\eta_{0}, \mathbf{k}\right)\right\rangle}{(2 \ell+1)^{2}}=\frac{\alpha^{2}}{8 \pi} \int_{0}^{\infty} d k k^{2}\left|\Omega_{B}(\mathbf{k})\right|^{2} j_{\ell}^{2}\left(k\left(\eta_{0}-\eta_{\mathrm{dec}}\right)\right) .
$$

In the case $n>-3 / 2$, substituting definition (16) and Eq. (13) in the above equation we have [40]

$$
C_{\ell}^{B} \simeq \frac{\alpha^{2} A^{2} k_{D}^{2 n+6}}{8(2 \pi)^{5}(2 n+3) \rho_{\mathrm{rel}}^{2}\left(k_{D} \eta_{0}\right)^{3}} \int_{0}^{k_{D} \eta_{0}} d x x^{2} j_{\ell}^{2}(x) \simeq \frac{\alpha^{2}}{512 \pi} \frac{(n+3)^{2}}{2 n+3} \frac{\left\langle B^{2}\right\rangle^{2}}{\rho_{\text {rel }}^{2}} \frac{1}{\left(k_{D} \eta_{0}\right)^{2}} \quad \text { for } n>-3 / 2,
$$

where $x=k \eta_{0}$, we have approximated $j_{\ell}\left(k\left(\eta_{0}-\eta_{\mathrm{dec}}\right)\right) \simeq j_{\ell}\left(k \eta_{0}\right)$ and we integrate only up to the upper cutoff $k_{D}$ since we are using the approximated expression Eq. (13) which is strictly valid only for $k \ll k_{D}$. For the second equality in the above equation, we have approximated the integral as given in Eq. (A2) of appendix A, since we have that $y=k_{D} \eta_{0} \gg 1$. We have also used Eq. (3) to express the result in terms of the mean squared magnetic field.

For $n=-2$, we use instead Eq. (14): substituting it in Eq. (19), we find

$$
C_{\ell}^{B} \simeq \frac{3 \alpha^{2} A^{2} k_{D}^{2}}{4096 \pi^{3} \rho_{\text {rel }}^{2}\left(k_{D} \eta_{0}\right)^{2}} \int_{0}^{k_{D} \eta_{0}} d x j_{\ell}^{2}(x) \simeq \frac{3 \pi \alpha^{2}}{8192} \frac{\left\langle B^{2}\right\rangle^{2}}{\rho_{\text {rel }}^{2}} \frac{1}{\left(k_{D} \eta_{0}\right)^{2}} \log \left(\frac{k_{D} \eta_{0}}{\ell}\right) \quad \text { for } n=-2
$$

where in the second equality we use the approximation given in Eq. (A4) of appendix A For more negative values of $n, n<-2$, in the absence of an exact expression, we use Eq. (15): the CMB spectrum becomes

$$
\begin{aligned}
C_{\ell}^{B} & \simeq \frac{3 \alpha^{2} A^{2} k_{D}^{2 n+6}}{1024 \pi^{5} \rho_{\mathrm{rel}}^{2}\left(k_{D} \eta_{0}\right)^{2 n+6}} \frac{n}{(2 n+3)(n+3)} \int_{0}^{k_{D} \eta_{0}} d x x^{2 n+5} j_{\ell}^{2}(x) \\
& \simeq \frac{3 \alpha^{2}}{4096 \sqrt{\pi}} \frac{n(n+3)}{(2 n+3)} \frac{\Gamma[-n-2]}{\Gamma[-n-3 / 2]} \frac{\left\langle B^{2}\right\rangle^{2}}{\rho_{\text {rel }}^{2}} \frac{1}{\left(k_{D} \eta_{0}\right)^{2 n+6}} \ell^{2 n+4} \quad \text { for } n<-2,
\end{aligned}
$$

where again for the second equality we have used Eq. A3 of appendix A. The slope in $\ell$ of this last expression is only approximatively recovered numerically, whereas it is perfectly recovered for Eqs. (20[21).

\section{CMB TEMPERATURE BISPECTRUM AT LARGE ANGULAR SCALES}

We want to evaluate the CMB angular bispectrum of the temperature anisotropy due to the Sachs Wolfe effect induced by the magnetic field energy density. The angular bispectrum is given by $\left\langle a_{\ell_{1} m_{1}} a_{\ell_{2} m_{2}} a_{\ell_{3} m_{3}}\right\rangle$, with the spherical harmonic expansion coefficients

$$
a_{\ell m}(\mathbf{x})=\int d \Omega_{\hat{n}} Y_{\ell m}^{*}(\hat{n} ; \hat{e}) \Theta^{(0)}(\mathbf{x}, \hat{n})
$$

where $Y_{\ell m}^{*}(\hat{n} ; \hat{e})$ is the spherical harmonic with respect to a basis where $\hat{e}$ is an arbitrary but fixed direction, and $\Theta^{(0)}(\mathbf{x}, \hat{n})$ is the scalar temperature perturbation at position $\mathbf{x}$ ( $\hat{n}$ is the direction of light propagation). Using the formalism developed in [30] one has

$$
\begin{array}{r}
\Theta^{(0)}(\mathbf{x}, \hat{n})=\int \frac{d^{3} k}{(2 \pi)^{3}} \Sigma_{\ell} \Theta_{\ell}^{(0)}\left(\eta_{0}, \mathbf{k}\right) G_{\ell}^{0}, \\
G_{\ell}^{0}=(-i)^{\ell} \sqrt{\frac{4 \pi}{2 \ell+1}} Y_{\ell 0}(\hat{n} ; \hat{k}) e^{i \mathbf{k} \cdot \mathbf{x}},
\end{array}
$$

with respect to a basis where $\hat{k}$ is fixed. Substituting the above expressions in Eq. (23), and changing basis accordingly (cf. [41]), one finds

$$
a_{\ell m}(\mathbf{x})=\frac{4 \pi(-i)^{\ell}}{2 \ell+1} \int \frac{d^{3} k}{(2 \pi)^{3}} \Theta_{\ell}^{(0)}\left(\eta_{0}, \mathbf{k}\right) e^{i \mathbf{k} \cdot \mathbf{x}} Y_{\ell m}^{*}(\hat{k} ; \hat{e})
$$

Therefore the angular bispectrum is given by (we place the observer in $\mathbf{x}=0$ )

$$
\begin{aligned}
\left\langle a_{\ell_{1} m_{1}} a_{\ell_{2} m_{2}} a_{\ell_{3} m_{3}}\right\rangle & =\frac{(4 \pi)^{3}(-i)^{\ell_{1}+\ell_{2}+\ell_{3}}}{\left(2 \ell_{1}+1\right)\left(2 \ell_{2}+1\right)\left(2 \ell_{3}+1\right)} \int \frac{d^{3} k d^{3} q d^{3} p}{(2 \pi)^{9}} Y_{\ell_{1} m_{1}}^{*}(\hat{k} ; \hat{e}) Y_{\ell_{2} m_{2}}^{*}(\hat{q} ; \hat{e}) Y_{\ell_{3} m_{3}}^{*}(\hat{p} ; \hat{e}) \\
& \times\left\langle\Theta_{\ell_{1}}^{(0)}\left(\eta_{0}, \mathbf{k}\right) \Theta_{\ell_{2}}^{(0)}\left(\eta_{0}, \mathbf{q}\right) \Theta_{\ell_{3}}^{(0)}\left(\eta_{0}, \mathbf{p}\right)\right\rangle .
\end{aligned}
$$


Remembering Eq. (18) and definition (16), we see that in order to proceed we need to evaluate the bispectrum of the magnetic energy density $\left\langle\rho_{B}(\mathbf{k}) \rho_{B}(\mathbf{q}) \rho_{B}(\mathbf{p})\right\rangle$.

\section{THE MAGNETIC ENERGY DENSITY BISPECTRUM}

From the expression of the magnetic energy density given in Eq. (10), we see that its bispectrum is given in terms of the six point correlation function of the magnetic field

$$
\left\langle\rho_{B}(\mathbf{k}) \rho_{B}(\mathbf{q}) \rho_{B}(\mathbf{p})\right\rangle=\frac{1}{(8 \pi)^{3}} \int \frac{d^{3} \tilde{k} d^{3} \tilde{q} d^{3} \tilde{p}}{(2 \pi)^{9}}\left\langle B_{i}(\tilde{\mathbf{k}}) B_{i}(\mathbf{k}-\tilde{\mathbf{k}}) B_{j}(\tilde{\mathbf{q}}) B_{j}(\mathbf{q}-\tilde{\mathbf{q}}) B_{l}(\tilde{\mathbf{p}}) B_{l}(\mathbf{p}-\tilde{\mathbf{p}})\right\rangle .
$$

Since the magnetic field is assumed to be a Gaussian variable, we can use Wick's theorem to decompose the six point correlation function into products of the magnetic field power spectrum. To compute the above expression, we then use the definition of the magnetic power spectrum Eq. (1) and the fact that $B_{i}^{*}(\mathbf{k})=B_{i}(-\mathbf{k})$. Of the total fifteen terms obtained using Wick's theorem, seven are irrelevant because they are one-point terms proportional to $\delta(\mathbf{k}), \delta(\mathbf{q})$ or $\delta(\mathbf{p})$, while eight terms survive. Each of the latter is the product of three power spectra, and consequently contains the product of three delta functions ( $c f$. Eq. (11)). Two delta functions can be integrated, while the remaining one reduces to $\delta(\mathbf{k}+\mathbf{q}+\mathbf{p})$, the homogeneity condition. Starting from Eq. (28), the final result depends on which of the variables of the triple integral remains. For example, performing the integration in $d^{3} \tilde{p}$ and $d^{3} \tilde{q}$ and leaving out $d^{3} \tilde{k}$, one obtains (appropriately renaming the mute indexes)

$$
\left\langle\rho_{B}(\mathbf{k}) \rho_{B}(\mathbf{q}) \rho_{B}(\mathbf{p})\right\rangle=\frac{1}{128 \pi^{3}} \delta(\mathbf{k}+\mathbf{p}+\mathbf{q}) \int d^{3} \tilde{k} P_{i j}(\tilde{\mathbf{k}}) P_{j l}(\mathbf{k}-\tilde{\mathbf{k}})\left[P_{i l}(\mathbf{q}+\tilde{\mathbf{k}})+P_{i l}(\mathbf{p}+\tilde{\mathbf{k}})\right],
$$

where for conciseness we have defined

$$
P_{i j}(\mathbf{k})=P_{B}(k)\left(\delta_{i j}-\hat{k}_{i} \hat{k}_{j}\right)
$$

(note that $P_{i j}(\mathbf{k})=P_{i j}(-\mathbf{k})$ ). On the other hand, integrating out $d^{3} \tilde{k}$ and $d^{3} \tilde{p}$ one obtains

$$
\left\langle\rho_{B}(\mathbf{k}) \rho_{B}(\mathbf{q}) \rho_{B}(\mathbf{p})\right\rangle=\frac{1}{128 \pi^{3}} \delta(\mathbf{k}+\mathbf{p}+\mathbf{q}) \int d^{3} \tilde{q} P_{i j}(\tilde{\mathbf{q}}) P_{j l}(\mathbf{q}-\tilde{\mathbf{q}})\left[P_{i l}(\mathbf{k}+\tilde{\mathbf{q}})+P_{i l}(\mathbf{p}+\tilde{\mathbf{q}})\right],
$$

while integrating out $d^{3} \tilde{q}$ and $d^{3} \tilde{k}$ one obtains

$$
\left\langle\rho_{B}(\mathbf{k}) \rho_{B}(\mathbf{q}) \rho_{B}(\mathbf{p})\right\rangle=\frac{1}{128 \pi^{3}} \delta(\mathbf{k}+\mathbf{p}+\mathbf{q}) \int d^{3} \tilde{p} P_{i j}(\tilde{\mathbf{p}}) P_{j l}(\mathbf{p}-\tilde{\mathbf{p}})\left[P_{i l}(\mathbf{k}+\tilde{\mathbf{p}})+P_{i l}(\mathbf{q}+\tilde{\mathbf{p}})\right] .
$$

This is just a consequence of the fact that the right hand side of Eq. (28) is not apparently symmetric under the exchange of $\mathbf{k}, \mathbf{q}$ and $\mathbf{p}$, contrary to the left hand side. Since the final result should be symmetric, we finally set:

$$
\begin{aligned}
\left\langle\rho_{B}(\mathbf{k}) \rho_{B}(\mathbf{q}) \rho_{B}(\mathbf{p})\right\rangle=\frac{\delta(\mathbf{k}+\mathbf{p}+\mathbf{q})}{384 \pi^{3}} & \left\{\int d^{3} \tilde{k} P_{i j}(\tilde{\mathbf{k}}) P_{j l}(\mathbf{k}-\tilde{\mathbf{k}})\left[P_{i l}(\mathbf{q}+\tilde{\mathbf{k}})+P_{i l}(\mathbf{p}+\tilde{\mathbf{k}})\right]\right. \\
& +\int d^{3} \tilde{k} P_{i j}(\tilde{\mathbf{k}}) P_{j l}(\mathbf{q}-\tilde{\mathbf{k}})\left[P_{i l}(\mathbf{k}+\tilde{\mathbf{k}})+P_{i l}(\mathbf{p}+\tilde{\mathbf{k}})\right] \\
& \left.+\int d^{3} \tilde{k} P_{i j}(\tilde{\mathbf{k}}) P_{j l}(\mathbf{p}-\tilde{\mathbf{k}})\left[P_{i l}(\mathbf{q}+\tilde{\mathbf{k}})+P_{i l}(\mathbf{k}+\tilde{\mathbf{k}})\right]\right\} .
\end{aligned}
$$

Using definitions (30) and (2) we have in all generality:

$$
P_{i j}(\mathbf{k}) P_{j l}(\mathbf{q}) P_{i l}(\mathbf{p})=A^{3} k^{n} p^{n} q^{n}\left[(\hat{k} \cdot \hat{q})^{2}+(\hat{k} \cdot \hat{p})^{2}+(\hat{q} \cdot \hat{p})^{2}-(\hat{k} \cdot \hat{q})(\hat{k} \cdot \hat{p})(\hat{q} \cdot \hat{p})\right] \text { if } k \leq k_{D}, q \leq k_{D}, p \leq k_{D},
$$

and zero else. Due to the complexity of the angular structure and of the integration boundary of the integrals in Eq. (33), we cannot derive an exact expression for $\left\langle\rho_{B}(\mathbf{k}) \rho_{B}(\mathbf{q}) \rho_{B}(\mathbf{p})\right\rangle$ which is valid for any configuration of $\mathbf{k}, \mathbf{q}, \mathbf{p}$. We can however give an analytical estimate of the result, which we present in the following.

We are interested in estimating the behaviour of the integrals in (33). From the expression in Eq. 34 it is clear that, depending on the value of the spectral index $n$, the integral could diverge in the infrared limit. On the other hand, 


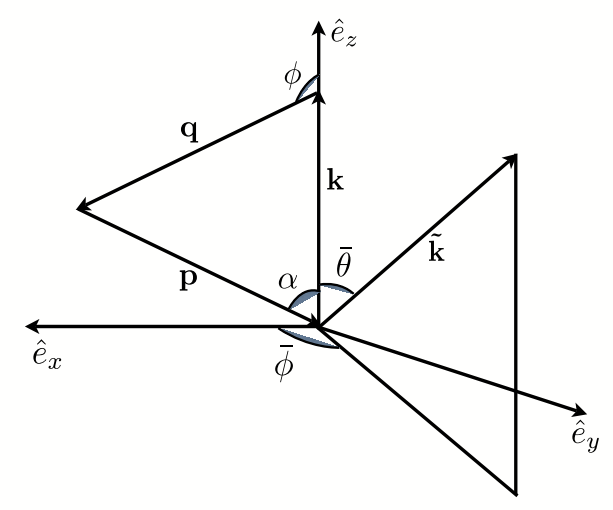

Figure 1: The geometrical configuration used to perform the integration: $\mathbf{k}, \mathbf{q}$ and $\mathbf{p}$ are free, while $\tilde{\mathbf{k}}$ is the integration wave-vector (see [25]).

the angular part always gives a finite contribution. We therefore neglect the angular part for the following estimate, and set

$$
\left\langle\rho_{B}(\mathbf{k}) \rho_{B}(\mathbf{q}) \rho_{B}(\mathbf{p})\right\rangle \simeq \frac{\delta(\mathbf{k}+\mathbf{p}+\mathbf{q})}{384 \pi^{3}} A^{3}\left\{\int d^{3} \tilde{k} \tilde{k}^{n}|\mathbf{k}-\tilde{\mathbf{k}}|^{n}\left[|\mathbf{q}+\tilde{\mathbf{k}}|^{n}+|\mathbf{p}+\tilde{\mathbf{k}}|^{n}\right]+\text { permutations }\right\} .
$$

To perform the above integration, following [25], we choose a basis with $\hat{e}_{z} \| \mathbf{k}$ and where the triangle formed by $\mathbf{k}, \mathbf{q}, \mathbf{p}$ lies in the plane perpendicular to $\hat{e}_{y}$, in $y=0$, see Fig. 1. We call $\phi$ the angle between $\mathbf{k}$ and $\mathbf{q}, \cos \phi=\hat{k} \cdot \hat{q}$, and $\alpha$ the angle between $\mathbf{k}$ and $-\mathbf{p}, \cos (\pi-\alpha)=\hat{k} \cdot \hat{p}$. The integration variable $\tilde{\mathbf{k}}$ has angles $\bar{\theta}$ with $\hat{e}_{z} \| \mathbf{k}$ and $\bar{\phi}$ with the plane identified by the triangle formed by $\mathbf{k}, \mathbf{q}, \mathbf{p}(c f$. Fig. 1). The angle between $\tilde{\mathbf{k}}$ and $\mathbf{q}$ is expressed in terms of the previously defined ones as

$$
\hat{\tilde{k}} \cdot \hat{q}=\sin \bar{\theta} \cos \bar{\phi} \sin \phi+\cos \bar{\theta} \cos \phi,
$$

and the one between $\tilde{\mathbf{k}}$ and $\mathbf{p}$ is

$$
\hat{\tilde{k}} \cdot \hat{p}=-(\sin \bar{\theta} \cos \bar{\phi} \sin \alpha+\cos \bar{\theta} \cos \alpha) .
$$

We remind that the boundaries of the integrals in (35) are defined by the condition that the momenta coming from the power spectrum are bounded by $k_{D}$ : in the first integral for example, the conditions are $\tilde{k} \leq k_{D},|\mathbf{k}-\tilde{\mathbf{k}}| \leq k_{D}$, $|\mathbf{q}+\tilde{\mathbf{k}}| \leq k_{D}$.

Let us first concentrate on the first integral of (35). For negative values of $n$, it has integrable divergences for $\tilde{\mathbf{k}} \rightarrow \mathbf{k}$ and for $\tilde{\mathbf{k}} \rightarrow-\mathbf{q}$. We approximate the total result by selecting only these angular configurations, which are the biggest contributions to the integral for negative $n$, and are at least representative of the total result for positive $n$. By doing so, and using the above reference system, the first integral of (35) becomes ( $\bar{\Omega}$ denotes the angular boundary)

$$
\begin{aligned}
\int d^{3} \tilde{k} \tilde{k}^{n}|\mathbf{k}-\tilde{\mathbf{k}}|^{n}|\mathbf{q}+\tilde{\mathbf{k}}|^{n} & =\int_{0}^{k_{D}} d \tilde{k} \tilde{k}^{n+2} \int_{\bar{\Omega}} d \Omega\left[k^{2}+\tilde{k}^{2}-2 k \tilde{k} \cos \bar{\theta}\right]^{n / 2}\left[q^{2}+\tilde{k}^{2}+2 q \tilde{k}(\sin \bar{\theta} \cos \bar{\phi} \sin \phi+\cos \bar{\theta} \cos \phi)\right]^{n / 2} \\
& \simeq 2 \pi \int_{0}^{k_{D}} d \tilde{k} \tilde{k}^{n+2}\left[|k-\tilde{k}|^{n}\left(q^{2}+\tilde{k}^{2}+2 q \tilde{k} \cos \phi\right)^{n / 2}+\left(k^{2}+\tilde{k}^{2}+2 k \tilde{k} \cos \phi\right)^{n / 2}|q-\tilde{k}|^{n}\right] \quad(38)
\end{aligned}
$$

where in the second equality we have accounted only for the two above mentioned angular configurations: the first term of the second equality is the contribution of the angular configuration $\tilde{\mathbf{k}} \rightarrow \mathbf{k}$, and therefore $\bar{\theta}=0$; the second one is the contribution of the angular configuration $\tilde{\mathbf{k}} \rightarrow-\mathbf{q}$, and therefore $\bar{\theta}=\pi-\phi$ and $\bar{\phi}=\pi$. We have inserted the factor $2 \pi$ to simulate the integration in $d \bar{\phi}$, which should be present at least in the first configuration. We repeat 
the same approximation scheme in each term of (35), to obtain finally

$$
\begin{aligned}
\left\langle\rho_{B}(\mathbf{k}) \rho_{B}(\mathbf{q}) \rho_{B}(\mathbf{p})\right\rangle \simeq & \frac{\delta(\mathbf{k}+\mathbf{p}+\mathbf{q})}{96 \pi^{2}} A^{3} \times \\
& \left\{\int_{0}^{k_{D}} d \tilde{k} \tilde{k}^{n+2}\left[|k-\tilde{k}|^{n}\left(q^{2}+\tilde{k}^{2}+2 q \tilde{k} \cos \phi\right)^{n / 2}+\left(k^{2}+\tilde{k}^{2}+2 k \tilde{k} \cos \phi\right)^{n / 2}|q-\tilde{k}|^{n}\right]\right. \\
& +\int_{0}^{k_{D}} d \tilde{k} \tilde{k}^{n+2}\left[|k-\tilde{k}|^{n}\left(p^{2}+\tilde{k}^{2}-2 p \tilde{k} \cos \alpha\right)^{n / 2}+\left(k^{2}+\tilde{k}^{2}-2 k \tilde{k} \cos \alpha\right)^{n / 2}|p-\tilde{k}|^{n}\right] \\
& \left.+\int_{0}^{k_{D}} d \tilde{k} \tilde{k}^{n+2}\left[|q-\tilde{k}|^{n}\left(p^{2}+\tilde{k}^{2}-2 p \tilde{k} \cos (\phi-\alpha)\right)^{n / 2}+\left(q^{2}+\tilde{k}^{2}-2 q \tilde{k} \cos (\phi-\alpha)\right)^{n / 2}|p-\tilde{k}|^{n}\right]\right\}
\end{aligned}
$$

Note that the terms in Eq. (35) which share the same wave-vectors collect two by two for the angular configurations considered (c.f. Eq. (33)). It is now possible to evaluate approximatively the above integrals. As already mentioned, the apparent divergence for negative $n$ is integrable. Assuming $k<q<k_{D}$, we approximate the first integral in the above expression as

$$
\begin{array}{r}
\int_{0}^{k_{D}} d \tilde{k} \tilde{k}^{n+2}\left[|k-\tilde{k}|^{n}\left(q^{2}+\tilde{k}^{2}+2 q \tilde{k} \cos \phi\right)^{n / 2}+\left(k^{2}+\tilde{k}^{2}+2 k \tilde{k} \cos \phi\right)^{n / 2}|q-\tilde{k}|^{n}\right] \simeq \\
2\left(q^{n} k^{n} \int_{0}^{k} d \tilde{k} \tilde{k}^{n+2}+q^{n} \int_{k}^{q} d \tilde{k} \tilde{k}^{2 n+2}+\int_{q}^{k_{D}} d \tilde{k} \tilde{k}^{3 n+2}\right) .
\end{array}
$$

We see that, under this approximation, the angular part plays no longer a role, and the result is the same for the two terms of the first line of the above equation.

Applying the same technique for each integral in Eq. (39), for the combination $k \leq q \leq p \leq k_{D}$ we find the total approximate behaviour:

$$
\begin{aligned}
\left\langle\rho_{B}(\mathbf{k}) \rho_{B}(\mathbf{q}) \rho_{B}(\mathbf{p})\right\rangle \simeq & \frac{\delta(\mathbf{k}+\mathbf{p}+\mathbf{q})}{48 \pi^{2}} A^{3} \times \\
& \left\{\frac{n}{(n+3)(2 n+3)} q^{n} k^{2 n+3}+\frac{n}{(3 n+3)(2 n+3)} q^{3 n+3}+\frac{k_{D}^{3 n+3}}{3 n+3}\right. \\
& +\frac{n}{(n+3)(2 n+3)} p^{n} k^{2 n+3}+\frac{n}{(3 n+3)(2 n+3)} p^{3 n+3}+\frac{k_{D}^{3 n+3}}{3 n+3} \\
& \left.+\frac{n}{(n+3)(2 n+3)} p^{n} q^{2 n+3}+\frac{n}{(3 n+3)(2 n+3)} p^{3 n+3}+\frac{k_{D}^{3 n+3}}{3 n+3}\right\} \quad \text { for } k \leq q \leq p \leq k_{D},
\end{aligned}
$$

while if $q \leq k \leq p$ we have to exchange $k$ and $q$ in the above expression, and so on with all the ordered permutations of the wave-numbers.

Observing Eq. (41), we can confirm what pointed out in 25], i.e. that there are two distinctive spectral regimes for the bispectrum. For flat and blue magnetic field spectra, with $n>-1$, the infrared limit $k \rightarrow 0$ of the bispectrum is white noise; Eq. (41) is in fact dominated by the constant terms $k_{D}^{3 n+3} /(3 n+3)$. On the other hand, for red magnetic field spectra $n<-1$, the bispectrum is divergent in the infrared limit. As we will see in the next sections, the divergence can go as $k^{2 n+3}$ or as $k^{3 n+3}$, depending on the wave-vector configuration. The same behaviour holds for the magnetic energy density power spectrum, but in this case the discriminating value is $n=-3 / 2$, and the infrared divergence for $n<-3 / 2$ goes as $k^{2 n+3}$ [27, 29] (in [27], it has been found that for the limiting value $n=-3 / 2$ the white noise spectrum acquires a logarithmic dependence on $k$ : this is the case also here for the corresponding limiting value $n=-1$ ). The above approximated result is valid only for $k, q$ and $p$ smaller than the magnetic upper cutoff $k_{D}$, while in general they do not need to satisfy this bound. As already mentioned in section [I] in [27] it has been found that the magnetic energy spectrum goes to zero at $k=2 k_{D}$, due to the convolution boundaries (c.f Eq. (12)). As we will see in the next sections, the same behaviour holds also for the bispectrum (this is verified exactly in the collinear configuration).

The above equation (41) is a general approximation to the magnetic field energy density bispectrum in the infrared limit. We now compare it with the result coming from a specific configuration of the wave-vectors, the collinear configuration, for which we have an exact result. We find that the above expression can be considered quite a good approximation to the true magnetic field bispectrum in the infrared limit. We also give explicit formulas for the squeezed and equilateral configurations, for which, however, we do not calculate the exact result. We find that the three configurations give a comparable white noise contribution for $n>-1$, while if $n<-1$ the collinear and equilateral configurations diverge in the infrared limit as $k^{3 n+3}$, while the squeezed one diverges as $k^{2 n+3}$. 


\section{A. Collinear configuration}

The collinear (or flattened) configuration is given by two equal wave-vectors, while the third one points in the opposite direction: for example, $\mathbf{p}=\mathbf{q}$ and $\mathbf{k}=-2 \mathbf{q}$. In this case, it is possible to calculate the bispectrum (33) exactly. The three permutations of $\mathbf{k}, \mathbf{q}, \mathbf{p}$ of this configuration should be present in the symmetric expression (33): this gives in the end

$$
\begin{aligned}
\left.\left\langle\rho_{B}(\mathbf{k}) \rho_{B}(\mathbf{q}) \rho_{B}(\mathbf{p})\right\rangle\right|_{\text {collinear }}=\frac{\delta(\mathbf{k}+\mathbf{p}+\mathbf{q})}{384 \pi^{3}} & \frac{2}{3} \int d^{3} \tilde{k} P_{i j}(\tilde{\mathbf{k}})\left\{P_{j l}\left(\frac{\mathbf{k}}{2}+\tilde{\mathbf{k}}\right)\left[P_{i l}(\mathbf{k}+\tilde{\mathbf{k}})+P_{i l}\left(\frac{\mathbf{k}}{2}-\tilde{\mathbf{k}}\right)\right]\right. \\
+ & \left.P_{j l}(\mathbf{k}-\tilde{\mathbf{k}}) P_{i l}\left(\frac{\mathbf{k}}{2}-\tilde{\mathbf{k}}\right)+\mathbf{k} \rightarrow \mathbf{p}+\mathbf{k} \rightarrow \mathbf{q}\right\} .
\end{aligned}
$$

Therefore, for the collinear case we find the following expression, using Eq. (34):

$$
\begin{aligned}
& \left.\left\langle\rho_{B}(\mathbf{k}) \rho_{B}(\mathbf{q}) \rho_{B}(\mathbf{p})\right\rangle\right|_{\text {collinear }}=\frac{\delta(\mathbf{k}+\mathbf{p}+\mathbf{q})}{576 \pi^{3}} A^{3} \times \\
& \left\{2 \int_{V_{1}} d^{3} \tilde{k} \tilde{k}^{n}\left|\frac{\mathbf{k}}{2}+\tilde{\mathbf{k}}\right|^{n}|\mathbf{k}+\tilde{\mathbf{k}}|^{n}\left[\frac{(\hat{\tilde{k}} \cdot \mathbf{k}+2 \tilde{k})^{2}}{4\left|\frac{\mathbf{k}}{2}+\tilde{\mathbf{k}}\right|^{2}}+\frac{(\hat{\tilde{k}} \cdot \mathbf{k}+\tilde{k})^{2}}{|\mathbf{k}+\tilde{\mathbf{k}}|^{2}}+\frac{\left(k^{2}+3 \tilde{\mathbf{k}} \cdot \mathbf{k}+2 \tilde{k} \tilde{k}^{2}\right)\left(k^{2}-(\tilde{\tilde{k}} \cdot \mathbf{k})^{2}\right)}{4\left|\frac{\mathbf{k}}{2}+\tilde{\mathbf{k}}\right|^{2}|\mathbf{k}+\tilde{\mathbf{k}}|^{2}}\right]\right. \\
& +\int_{V_{2}} d^{3} \tilde{k} \tilde{k}^{n}\left|\frac{\mathbf{k}}{2}+\tilde{\mathbf{k}}\right|^{n}\left|\frac{\mathbf{k}}{2}-\tilde{\mathbf{k}}\right|^{n}\left[\frac{(\hat{\tilde{k}} \cdot \mathbf{k}+2 \tilde{k})^{2}}{4\left|\frac{\mathbf{k}}{2}+\tilde{\mathbf{k}}\right|^{2}}+\frac{(\hat{\tilde{k}} \cdot \mathbf{k}-2 \tilde{k})^{2}}{4\left|\frac{\mathbf{k}}{2}-\tilde{\mathbf{k}}\right|^{2}}+\frac{\left(k^{2}-4 \tilde{k}^{2}\right)\left(k^{2}-(\hat{\tilde{k}} \cdot \mathbf{k})^{2}\right)}{16\left|\frac{\mathbf{k}}{2}+\tilde{\mathbf{k}}\right|^{2}\left|\frac{\mathbf{k}}{2}-\tilde{\mathbf{k}}\right|^{2}}\right] \\
& +\mathbf{k} \rightarrow \mathbf{p}+\mathbf{k} \rightarrow \mathbf{q}\}
\end{aligned}
$$

where $V_{1}$ denotes the volume given by the three conditions

$$
\begin{aligned}
\tilde{k} & \leq k_{D} \\
|\mathbf{k} / 2+\tilde{\mathbf{k}}| & \leq k_{D} \\
|\mathbf{k}+\tilde{\mathbf{k}}| & \leq k_{D},
\end{aligned}
$$

and $V_{2}$ is given by the conditions

$$
\begin{aligned}
\tilde{k} & \leq k_{D} \\
|\mathbf{k} / 2+\tilde{\mathbf{k}}| & \leq k_{D} \\
|\mathbf{k} / 2-\tilde{\mathbf{k}}| & \leq k_{D} .
\end{aligned}
$$

The last term of Eq. 42, $P_{i j}(\tilde{\mathbf{k}}) P_{j l}(\mathbf{k}-\tilde{\mathbf{k}}) P_{i l}\left(\frac{\mathbf{k}}{2}-\tilde{\mathbf{k}}\right)$, becomes equal to the first one by changing $\tilde{\mathbf{k}}$ to $-\tilde{\mathbf{k}}$ and the integration volume accordingly.

It is possible to calculate Eq. (43) exactly for the selected values of the spectral index $n=2$ and $n=-2$. This is due to the fact that, in this configuration, the integration over the angle $\bar{\phi}$ becomes trivial (c.f. Fig. 1): since $\mathbf{p}=\mathbf{q}$ and $\mathbf{k}=-2 \mathbf{q}$, the integrands in Eq. (43) depend only on $\cos \bar{\theta}$ and the boundaries given by $V_{1}$ and $V_{2}$ can be made explicit with little difficulty. The details of the calculation are given in Appendix B while the result is shown in Fig. 2. In the case $n=-2$ the calculation is quite involved, therefore we have evaluated only the infrared part, up to $k \leq k_{D} / 2$. On the other hand, the case $n=2$ is simpler, and in this case we found a general, exact expression. This expression confirms that the cutoff of the bispectrum is at $k=2 k_{D}$, as we would expect from the analysis of the spectrum (see Fig. 3), and as can be viewed easily from the last inequality of the boundary conditions of $V_{1}$, $|\mathbf{k}+\tilde{\mathbf{k}}| \leq k_{D}$, which shows that the maximal allowed value for $k$ is $2 k_{D}$.

Knowing the exact result, we can test the goodness of the approximation given in the last section at least in this configuration. Reducing the general result of Eq. (41) in the collinear configuration, we find:

$$
\begin{aligned}
\left.\left\langle\rho_{B}(\mathbf{k}) \rho_{B}(\mathbf{q}) \rho_{B}(\mathbf{p})\right\rangle\right|_{\text {collinear }} \simeq & \frac{\delta(\mathbf{k}+\mathbf{p}+\mathbf{q})}{144 \pi^{2}} A^{3}\left\{\frac{n}{2^{3 n+3}(2 n+3)}\left(\frac{2^{n+1}+1}{n+3}+\frac{2^{3 n+4}+1}{3 n+3}\right) k^{3 n+3}+\frac{k_{D}^{3 n+3}}{n+1}\right. \\
& +k \rightarrow p+k \rightarrow q\} .
\end{aligned}
$$

Given that $k \leq k_{D}$, if $n<-1$, this expression is divergent for $k \rightarrow 0$ as $k^{3 n+3}$, while for $n>-1$, is it white noise. Consequently, the case $n=-2$ exhibits a divergent behaviour as $k^{-3}$, while the case $n=2$ is regular, as can be seen 

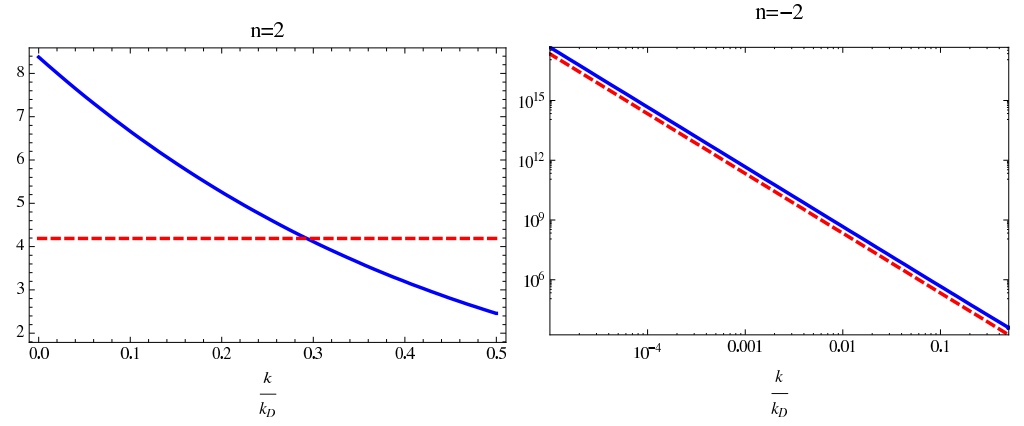

Figure 2: The magnetic field bispectrum in the collinear configuration $\mathbf{p}=\mathbf{q}=-\mathbf{k} / 2$, normalised by the quantity $A^{3} k_{D}^{3 n+3} /\left(576 \pi^{3}\right)$, as a function of $k / k_{D}$, for $n=2$ (left plot) and $n=-2$ (right plot). We only show the infrared region $k \leq k_{D} / 2$. The blue, solid line is the exact result, while the red, dashed line the approximation given in Eq. (46).
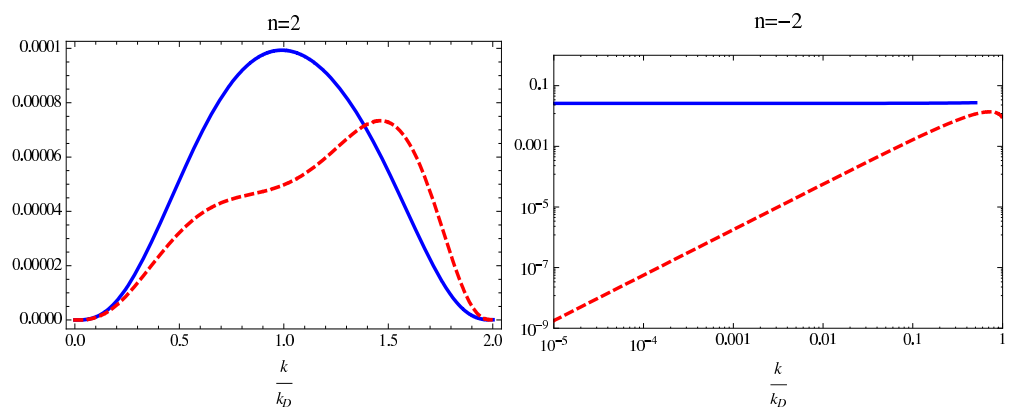

Figure 3: The magnetic field bispectrum in the collinear configuration $\mathbf{p}=\mathbf{q}=-\mathbf{k} / 2$ (blue, solid) and the magnetic field spectrum to the $3 / 2$ (red, dashed), both multiplied by the phase space density $k^{3}$, as a function of $k / k_{D}$ for $n=2$ and $n=-2$. Note that in the $n=-2$ case, we only calculated the bispectrum up to $k=k_{D} / 2$, while the spectrum is known up to $k=k_{D}$.

in Fig. 2, where the true and approximated result are compared. In the regular $n=2$ case, the bispectrum is not pure white noise but shows a mild dependence on $k$ : our approximation does not capture this dependence, but only the infrared white noise behaviour. In both cases, our approximation underestimates the true result by a factor of two.

In Fig. 3, we compare the exact result of the bispectrum in the collinear configuration with the magnetic spectrum to the power $3 / 2$, for $n=2$ and $n=-2$, both multiplied by the phase space density $\left(k / k_{D}\right)^{3}$. For $n=2$, they are of the same order of magnitude, as one would expect. For $n=-2$, the bispectrum goes as $k^{3 n+3}$, while the spectrum as $k^{2 n+3}$. The spectrum approaches the bispectrum amplitude as $k$ grows, however, the exact bispectrum has been calculated only for $k \leq k_{D} / 2$, and this is the region shown in the plot.

\section{B. Squeezed configuration}

In the squeezed configuration one wave-vector goes to zero while the other two are equal but opposite in direction. Expliciting the case in which $\mathbf{q} \simeq 0, \mathbf{k}=-\mathbf{p}$, from Eq. (33) we have

$$
\begin{aligned}
\left.\left\langle\rho_{B}(\mathbf{k}) \rho_{B}(\mathbf{q}) \rho_{B}(\mathbf{p})\right\rangle\right|_{\text {squeezed }}=\frac{\delta(\mathbf{k}+\mathbf{p}+\mathbf{q})}{384 \pi^{3}} & \frac{1}{3} \int d^{3} \tilde{k} P_{i j}(\tilde{\mathbf{k}})\left\{P_{j l}(\mathbf{k}-\tilde{\mathbf{k}})\left[P_{i l}(\mathbf{q}+\tilde{\mathbf{k}})+P_{i l}(\mathbf{k}-\tilde{\mathbf{k}})\right]\right. \\
& +P_{j l}(\mathbf{q}-\tilde{\mathbf{k}})\left[P_{i l}(\mathbf{k}-\tilde{\mathbf{k}})+P_{i l}(\mathbf{k}+\tilde{\mathbf{k}})\right] \\
& +P_{j l}(\mathbf{k}+\tilde{\mathbf{k}})\left[P_{i l}(\mathbf{k}+\tilde{\mathbf{k}})+P_{i l}(\mathbf{q}+\tilde{\mathbf{k}})\right] \\
& +(\mathbf{q} \rightarrow \mathbf{p} \simeq 0, \mathbf{k} \rightarrow \mathbf{q})+(\mathbf{q} \rightarrow \mathbf{k} \simeq 0, \mathbf{k} \rightarrow \mathbf{p})\}
\end{aligned}
$$


Using Eq. 34, and grouping the terms which are mutually equal, we find:

$$
\begin{aligned}
& \left.\left\langle\rho_{B}(\mathbf{k}) \rho_{B}(\mathbf{q}) \rho_{B}(\mathbf{p})\right\rangle\right|_{\text {squeezed }}=\frac{\delta(\mathbf{k}+\mathbf{p}+\mathbf{q})}{384 \pi^{3}} A^{3} \times \\
& \frac{2}{3}\left\{\int_{V_{1}} d^{3} \tilde{k} \tilde{k}^{n}|\mathbf{k}-\tilde{\mathbf{k}}|^{n}|\mathbf{q}+\tilde{\mathbf{k}}|^{n}\left[\frac{(\hat{\tilde{k}} \cdot \mathbf{k}-\tilde{k})^{2}}{|\mathbf{k}-\tilde{\mathbf{k}}|^{2}}+\frac{(\hat{\tilde{k}} \cdot \mathbf{q}+\tilde{k})^{2}}{|\mathbf{q}+\tilde{\mathbf{k}}|^{2}}+\frac{\left(\mathbf{k} \cdot \mathbf{q}-\mathbf{k} \cdot \tilde{\mathbf{k}}+\mathbf{q} \cdot \tilde{\mathbf{k}}-\tilde{k}{ }^{2}\right)[\mathbf{k} \cdot \mathbf{q}-(\hat{\tilde{k}} \cdot \mathbf{k})(\tilde{\tilde{k}} \cdot \mathbf{q})]}{|\mathbf{k}-\tilde{\mathbf{k}}|^{2}|\mathbf{q}+\tilde{\mathbf{k}}|^{2}}\right]\right. \\
& +\int_{V_{2}} d^{3} \tilde{k} \tilde{k}^{n}|\mathbf{k}-\tilde{\mathbf{k}}|^{2 n}\left[2 \frac{(\hat{\tilde{k}} \cdot \mathbf{k}-\tilde{k})^{2}}{|\mathbf{k}-\tilde{\mathbf{k}}|^{2}}+\frac{\left(k^{2}-2 \mathbf{k} \cdot \tilde{k}+\tilde{k}^{2}\right)\left[k^{2}-(\hat{\tilde{k}} \cdot \mathbf{k})^{2}\right]}{|\mathbf{k}-\tilde{\mathbf{k}}|^{4}}\right] \\
& +\int_{V_{3}} d^{3} \tilde{k} \tilde{k}^{n}|\mathbf{q}-\tilde{\mathbf{k}}|^{n}|\mathbf{k}-\tilde{\mathbf{k}}|^{n}\left[\frac{(\tilde{\tilde{k}} \cdot \mathbf{k}-\tilde{k})^{2}}{|\mathbf{k}-\tilde{\mathbf{k}}|^{2}}+\frac{(\tilde{\tilde{k}} \cdot \mathbf{q}-\tilde{k})^{2}}{|\mathbf{q}-\tilde{\mathbf{k}}|^{2}}+\frac{\left(\mathbf{k} \cdot \mathbf{q}-\mathbf{k} \cdot \tilde{\mathbf{k}}-\mathbf{q} \cdot \tilde{\mathbf{k}}+\tilde{k}^{2}\right)[\mathbf{k} \cdot \mathbf{q}-(\tilde{\tilde{k}} \cdot \mathbf{k})(\hat{\tilde{k}} \cdot \mathbf{q})]}{|\mathbf{k}-\tilde{\mathbf{k}}|^{2}|\mathbf{q}-\tilde{\mathbf{k}}|^{2}}\right] \\
& +(\mathbf{q} \rightarrow \mathbf{p} \simeq 0, \mathbf{k} \rightarrow \mathbf{q})+(\mathbf{q} \rightarrow \mathbf{k} \simeq 0, \mathbf{k} \rightarrow \mathbf{p})\},
\end{aligned}
$$

where $V_{1}$ is given by the conditions

$$
\begin{aligned}
\tilde{k} & \leq k_{D} \\
|\mathbf{k}-\tilde{\mathbf{k}}| & \leq k_{D} \\
|\mathbf{q}+\tilde{\mathbf{k}}| & \leq k_{D},
\end{aligned}
$$

$V_{2}$ by the conditions

$$
\begin{aligned}
\tilde{k} & \leq k_{D} \\
|\mathbf{k}-\tilde{\mathbf{k}}| & \leq k_{D},
\end{aligned}
$$

and $V_{3}$ by the conditions

$$
\begin{aligned}
\tilde{k} & \leq k_{D} \\
|\mathbf{q}-\tilde{\mathbf{k}}| & \leq k_{D} \\
|\mathbf{k}-\tilde{\mathbf{k}}| & \leq k_{D} .
\end{aligned}
$$

We do not have an exact calculation of the bispectrum in the squeezed configuration. This is due to the fact that, contrary to the collinear case, the integration over the angle $\bar{\phi}$ is not trivial (c.f. Fig. 1). For example, for the case $\mathbf{q} \simeq 0, \mathbf{k}=-\mathbf{p}$, the angle $\phi \rightarrow \pi / 2$ : therefore, taking for example the first integral in Eq. (48), we see that it contains the term $\left(q^{2}+\tilde{k}^{2}+2 \tilde{k} q \sin \bar{\theta} \cos \bar{\phi}\right)^{n / 2}$, and the integration boundary over $\bar{\theta}$ and $\bar{\phi}$ given by $V_{1}$ becomes very complicated. Having already an estimation of the goodness of our approximation in the collinear case, we do not dwell on the calculation for the squeezed configuration, and use only the approximated formula. Observing the boundary conditions given by the integration volumes $V_{1}, V_{2}$ and $V_{3}$ we can however confirm that, also in the squeezed configuration, the bispectrum goes to zero at $k=q=2 k_{D}$, since these are the maximally allowed values for the wave-numbers.

We reduce the general expression given in Eq. (41) in the squeezed configuration, and find:

$$
\begin{aligned}
\left.\left\langle\rho_{B}(\mathbf{k}) \rho_{B}(\mathbf{q}) \rho_{B}(\mathbf{p})\right\rangle\right|_{\text {squeezed }} \simeq & \frac{\delta(\mathbf{k}+\mathbf{p}+\mathbf{q})}{144 \pi^{2}} A^{3}\left\{\frac{2 n}{(n+3)(2 n+3)} q^{2 n+3} k^{n}+\frac{6 n(n+2)}{(3 n+3)(2 n+3)(n+3)} k^{3 n+3}\right. \\
& \left.+\frac{k_{D}^{3 n+3}}{n+1}+(q \rightarrow p \simeq 0, k \rightarrow q)+(q \rightarrow k \simeq 0, k \rightarrow p)\right\} .
\end{aligned}
$$

For $n>-1$, the resulting white noise plateau has the same amplitude as in the collinear case. However, for $n<-1$ the divergence for $q \rightarrow 0$ is $q^{2 n+3}$ : therefore, it is weaker than in the collinear case, and reaches the collinear behaviour $q^{3 n+3}$ only in the limit $k \rightarrow q \rightarrow 0$. 


\section{Equilateral configuration}

In the equilateral configuration the wave-vectors form an equilateral triangle. With $\mathbf{q}=k \hat{q}$, and $\mathbf{p}=k \hat{p}$, using Eq. (33) and regrouping the equal terms one gets

$$
\begin{aligned}
\left.\left\langle\rho_{B}(\mathbf{k}) \rho_{B}(\mathbf{q}) \rho_{B}(\mathbf{p})\right\rangle\right|_{\text {equilateral }} & =\frac{\delta(\mathbf{k}+\mathbf{p}+\mathbf{q})}{384 \pi^{3}} \frac{2}{3} \int d^{3} \tilde{k} P_{i j}(\tilde{\mathbf{k}})\left\{P_{j l}(\mathbf{k}-\tilde{\mathbf{k}})\left[P_{i l}(k \hat{q}+\tilde{\mathbf{k}})+P_{i l}(k \hat{p}+\tilde{\mathbf{k}})\right]\right. \\
& \left.+P_{j l}(k \hat{q}-\tilde{\mathbf{k}}) P_{i l}(k \hat{p}+\tilde{\mathbf{k}})+(\mathbf{k} \rightarrow \mathbf{q}, k \hat{q} \rightarrow q \hat{k}, k \hat{p} \rightarrow q \hat{p})+(\mathbf{k} \rightarrow \mathbf{p}, k \hat{q} \rightarrow p \hat{q}, k \hat{p} \rightarrow p \hat{k})\right\}
\end{aligned}
$$

Using Eq. (34), we can rewrite the above expression explicitly as

$$
\begin{aligned}
& \left.\left\langle\rho_{B}(\mathbf{k}) \rho_{B}(\mathbf{q}) \rho_{B}(\mathbf{p})\right\rangle\right|_{\text {equilateral }}=\frac{\delta(\mathbf{k}+\mathbf{p}+\mathbf{q})}{384 \pi^{3}} A^{3} \times \\
& \frac{2}{3}\left\{\int_{V_{1}} d^{3} \tilde{k} \tilde{k}^{n}|\mathbf{k}-\tilde{\mathbf{k}}|^{n}|k \hat{q}+\tilde{\mathbf{k}}|^{n}\left[\frac{(\hat{\tilde{k}} \cdot \mathbf{k}-\tilde{k})^{2}}{|\mathbf{k}-\tilde{\mathbf{k}}|^{2}}+\frac{(\hat{\tilde{k}} \cdot k \hat{q}+\tilde{k})^{2}}{|k \hat{q}+\tilde{\mathbf{k}}|^{2}}+\frac{\left.(\mathbf{k} \cdot k \hat{q}-\mathbf{k} \cdot \tilde{\mathbf{k}}+k \hat{q} \cdot \tilde{\mathbf{k}}-\tilde{k})^{2}\right)[\mathbf{k} \cdot k \hat{q}-(\hat{\tilde{k}} \cdot \mathbf{k})(\tilde{\tilde{k}} \cdot k \hat{q})]}{|\mathbf{k}-\tilde{\mathbf{k}}|^{2}|k \hat{q}+\tilde{\mathbf{k}}|^{2}}\right]\right. \\
& +\int_{V_{2}} d^{3} \tilde{k} \tilde{k}^{n}|\mathbf{k}-\tilde{\mathbf{k}}|^{n}|k \hat{p}+\tilde{\mathbf{k}}|^{n}\left[\frac{(\hat{\tilde{k}} \cdot \mathbf{k}-\tilde{k})^{2}}{|\mathbf{k}-\tilde{\mathbf{k}}|^{2}}+\frac{(\hat{\tilde{k}} \cdot k \hat{p}+\tilde{k})^{2}}{|k \hat{p}+\tilde{\mathbf{k}}|^{2}}+\frac{\left.(\mathbf{k} \cdot k \hat{p}-\mathbf{k} \cdot \tilde{\mathbf{k}}+k \hat{p} \cdot \tilde{\mathbf{k}}-\tilde{k})^{2}\right)[\mathbf{k} \cdot k \hat{p}-(\hat{\tilde{k}} \cdot \mathbf{k})(\hat{\tilde{k}} \cdot k \hat{p})]}{|\mathbf{k}-\tilde{\mathbf{k}}|^{2}|k \hat{p}+\tilde{\mathbf{k}}|^{2}}\right] \\
& +\int_{V_{3}} d^{3} \tilde{k} \tilde{k}^{n}|k \hat{q}-\tilde{\mathbf{k}}|^{n}|k \hat{p}+\tilde{\mathbf{k}}|^{n}\left[\frac{(\hat{\tilde{k}} \cdot k \hat{q}-\tilde{k})^{2}}{|k \hat{q}-\tilde{\mathbf{k}}|^{2}}+\frac{(\hat{\tilde{k}} \cdot k \hat{p}+\tilde{k})^{2}}{|k \hat{p}+\tilde{\mathbf{k}}|^{2}}+\frac{\left(k \hat{q} \cdot k \hat{p}-k \hat{q} \cdot \tilde{\mathbf{k}}+k \hat{p} \cdot \tilde{\mathbf{k}}-\tilde{k}{ }^{2}\right)[k \hat{q} \cdot k \hat{p}-(\hat{\tilde{k}} \cdot k \hat{q})(\hat{\tilde{k}} \cdot k \hat{p})]}{|k \hat{q}-\tilde{\mathbf{k}}|^{2}|k \hat{p}+\tilde{\mathbf{k}}|^{2}}\right] \\
& +(\mathbf{k} \rightarrow \mathbf{q}, k \hat{q} \rightarrow q \hat{k}, k \hat{p} \rightarrow q \hat{p})+(\mathbf{k} \rightarrow \mathbf{p}, k \hat{q} \rightarrow p \hat{q}, k \hat{p} \rightarrow p \hat{k})\}
\end{aligned}
$$

where again $V_{1}$ is given by the conditions

$$
\begin{aligned}
\tilde{k} & \leq k_{D} \\
|\mathbf{k}-\tilde{\mathbf{k}}| & \leq k_{D} \\
|k \hat{q}+\tilde{\mathbf{k}}| & \leq k_{D},
\end{aligned}
$$

and similarly for $V_{2}$ and $V_{3}$. In this case as well, we cannot solve the above integrals exactly. Like in the squeezed configuration, the integration in $d \bar{\phi}$ is non-trivial, since $\phi=2 \pi / 3$ and, for example, the first integral of Eq. (54) contains terms like $\left(k^{2}+\tilde{k}^{2}+2 \tilde{k} k\left(\frac{1}{2} \sin \bar{\theta} \cos \bar{\phi}-\frac{1}{2} \cos \bar{\theta}\right)\right)^{n / 2}$. We therefore use the approximated expression in Eq. (41), which gives simply:

$$
\left.\left\langle\rho_{B}(\mathbf{k}) \rho_{B}(\mathbf{q}) \rho_{B}(\mathbf{p})\right\rangle\right|_{\text {equilateral }} \simeq \frac{\delta(\mathbf{k}+\mathbf{p}+\mathbf{q})}{144 \pi^{2}} A^{3}\left\{\frac{6 n}{(n+3)(3 n+3)} k^{3 n+3}+\frac{k_{D}^{3 n+3}}{n+1}+(k \rightarrow q)+(k \rightarrow p)\right\} .
$$

For $n>-1$, we find again a white noise plateau of the same amplitude as in the other configurations; for $n<-1$ the divergence for $k \rightarrow 0$ is the same as in the collinear case. At first sight, this result might not seem correct: in the collinear case, in fact, by definition the wave-vectors are collinear and therefore the limits $\tilde{\mathbf{k}} \rightarrow \mathbf{k}$ and $\tilde{\mathbf{k}} \rightarrow-\mathbf{q}$ collapse into a single wave-vector configuration. In the equilateral case, on the other hand, they do not: we would therefore naively expect the same infrared behaviour of the squeezed configuration. However, the infrared divergence occurs for $k=q=p \rightarrow 0$, and in this limit $\tilde{\mathbf{k}} \rightarrow \mathbf{k}$ and $\tilde{\mathbf{k}} \rightarrow-\mathbf{q}$ are no longer distinct. Therefore, we do expect a $k^{3 n+3}$ behaviour also in the equilateral case, equivalent to what we find in the collinear case and also in the squeezed one when we let not only $q$, but also $k \rightarrow 0$ ( $c . f$ Eq. (52)).

We can conclude that, although it neglects the angles, the approximation in Eq. (41) does recover the correct behaviour of the bispectrum in the analysed configurations. However, neglecting the angles certainly introduces an inaccuracy, because one does not account precisely for the weight with which the different configurations contribute to the total result. We were able to compare the approximated result with the exact one only in the collinear configuration, and we found an underestimation of a factor of two both for negative and positive spectral indexes. However, this does not ensure that the total, exact bispectrum is altogether only a factor of two higher than what given in Eq. (41), neither that it has exactly the same dependence on wave-numbers when we significantly deviate from the infrared limit. 


\section{THE CMB BISPECTRUM}

Given the magnetic energy density bispectrum $\left\langle\rho_{B}(\mathbf{k}) \rho_{B}(\mathbf{q}) \rho_{B}(\mathbf{p})\right\rangle$, we can now evaluate the CMB bispectrum Eq. (27). We use the approximated magnetic energy bispectrum Eq. (41),

$$
\begin{aligned}
\left\langle\rho_{B}(\mathbf{k}) \rho_{B}(\mathbf{q}) \rho_{B}(\mathbf{p})\right\rangle \simeq & \delta(\mathbf{k}+\mathbf{p}+\mathbf{q}) \frac{A^{3} k_{D}^{3 n+3}}{48 \pi^{2}} \mathcal{I}(K, Q, P) \\
\mathcal{I}(K, Q, P)= & \frac{n}{(n+3)(2 n+3)} Q^{n} K^{2 n+3}+\frac{n}{(3 n+3)(2 n+3)} Q^{3 n+3}+\frac{n}{(n+3)(2 n+3)} P^{n} K^{2 n+3} \\
& +\frac{n}{(3 n+3)(2 n+3)} P^{3 n+3}+\frac{n}{(n+3)(2 n+3)} P^{n} Q^{2 n+3}+\frac{n}{(3 n+3)(2 n+3)} P^{3 n+3} \\
& +\frac{1}{n+1} \quad \text { for } K \leq Q \leq P \leq 1,
\end{aligned}
$$

where $K=k / k_{D}$ and so on denote normalised wave-numbers. We want to estimate the reduced bispectrum $b_{\ell_{1} \ell_{2} \ell_{3}}$ introduced in [42]

$$
\left\langle a_{\ell_{1} m_{1}} a_{\ell_{2} m_{2}} a_{\ell_{3} m_{3}}\right\rangle=\mathcal{G}_{\ell_{1} \ell_{2} \ell_{3}}^{m_{1} m_{2} m_{3}} b_{\ell_{1} \ell_{2} \ell_{3}}
$$

where $\mathcal{G}_{\ell_{1} \ell_{2} \ell_{3}}^{m_{1} m_{2} m_{3}}$ is the Gaunt integral. We use the procedure described in [43]: starting from Eq. (27), substituting in it Eq. (18) and Eq. (16), and using expression (58) for the source, we find:

$$
\begin{aligned}
b_{\ell_{1} \ell_{2} \ell_{3}}= & \frac{\pi \alpha^{3} A^{3} k_{D}^{3 n+9}}{6 \rho_{\mathrm{rel}}^{3}} \int_{0}^{\infty} d x x^{2} \int_{0}^{1} d K K^{2} \int_{0}^{1} d Q Q^{2} \int_{0}^{1} d P P^{2} j_{\ell_{1}}(K y) j_{\ell_{1}}(K x) j_{\ell_{2}}(Q y) \\
& j_{\ell_{2}}(Q x) j_{\ell_{3}}(P y) j_{\ell_{3}}(P x) \mathcal{I}(K, Q, P),
\end{aligned}
$$

where $y=k_{D} \eta_{0}$ and $x=k_{D} r$, and $r$ comes from the decomposition of the delta function in (58) (see [43]). Using the definition of the bispectrum $\mathcal{I}(K, Q, P)$ given in (58), the above equation becomes

$$
\begin{aligned}
b_{\ell_{1} \ell_{2} \ell_{3}} & =\frac{\pi \alpha^{3} A^{3} k_{D}^{3 n+9}}{36 \rho_{\mathrm{rel}}^{3}} \int_{0}^{\infty} d x x^{2} \int_{0}^{1} d K K^{2} j_{\ell_{1}}(K y) j_{\ell_{1}}(K x) \int_{0}^{K} d Q Q^{2} j_{\ell_{2}}(Q y) j_{\ell_{2}}(Q x) \int_{0}^{Q} d P P^{2} j_{\ell_{3}}(P y) j_{\ell_{3}}(P x) \\
& \times\left\{a(n)\left[K^{n} Q^{2 n+3}+K^{n} P^{2 n+3}+Q^{n} P^{2 n+3}\right]+b(n)\left[2 K^{3 n+3}+Q^{3 n+3}\right]+c(n)\right\} \\
& + \text { permutations }
\end{aligned}
$$

where $a(n)=n /(n+3) /(2 n+3), b(n)=n /(3 n+3) /(2 n+3), c(n)=1 /(n+1)$, and one adds the six ordered permutations of $K, Q$ and $P$ which entail permutations of $\ell_{1}, \ell_{2}, \ell_{3}$.

In order to estimate the bispectrum, we substitute the upper boundaries in Eq. (61) with the interval [0,1] in all the integrals over the momenta, since the Bessel functions peak at very low momentum: $j_{\ell_{3}}(P y)$ peaks at $P \simeq \ell_{3} / y$, and $y \gg 1$. Because of the form of the source $\mathcal{I}(K, Q, P)$, in (61) at least one integral over the momentum is not influenced by the source. Following [44], for each of these integral we use the approximation (cf. Eq. 6.512 of [45])

$$
\int_{0}^{1} d P P^{2} j_{\ell_{3}}(P y) j_{\ell_{3}}(P x) \sim \frac{1}{4} \frac{\delta(y-x)}{x^{2}}
$$

we then solve the integral in $d x$ using the delta function and obtain for the first term for example,

$$
\frac{a(n)}{4} \int_{0}^{1} d K K^{n+2} j_{\ell_{1}}^{2}(K y) \int_{0}^{1} d Q Q^{2 n+5} j_{\ell_{2}}^{2}(Q y)
$$

and so on. Approximate expressions for this kind of integrals are discussed in appendix $\mathrm{A}$

If $n>-1$, in Eq. (61) we retain only the white noise term $c(n)$. All permutations give the same result in this case, and we find finally

$$
b_{\ell_{1} \ell_{2} \ell_{3}} \simeq \frac{\pi^{7} \alpha^{3}}{96} \frac{(n+3)^{3}}{n+1} \frac{\left\langle B^{2}\right\rangle^{3}}{\rho_{\text {rel }}^{3}} \frac{1}{\left(k_{D} \eta_{0}\right)^{4}}, \quad \text { for } n>-1
$$

Values of the spectral index $n<-1$, for which the source is not pure white noise, are a bit more involved. As in the spectrum case ( $c f$. section III), we cannot give a general expression valid for every $n<-1$, since the way to 
approximate integrals like those in (63) depends on the actual value of the power law exponent. Therefore, we give explicit expressions only for two values of the spectral index: $n=-2$, and $n \rightarrow-3$. Fixing the spectral index to $n=-2$, one finds

$$
\begin{aligned}
b_{\ell_{1} \ell_{2} \ell_{3}} & \simeq \frac{\pi^{8} \alpha^{3}}{288} \frac{\left\langle B^{2}\right\rangle^{3}}{\rho_{\text {rel }}^{3}} \frac{1}{\left(k_{D} \eta_{0}\right)^{3}}\left\{\frac{1}{\ell_{1}}\left[\log \left(\frac{k_{D} \eta_{0}}{\sqrt{\ell_{2}} \sqrt{\ell_{3}}}\right)-\frac{2 k_{D} \eta_{0}}{3 \pi} \frac{1}{\ell_{1}}\right]+\frac{1}{\ell_{2}}\left[\frac{1}{2} \log \left(\frac{k_{D} \eta_{0}}{\ell_{3}}\right)-\frac{k_{D} \eta_{0}}{3 \pi} \frac{1}{\ell_{2}}\right]\right\} \\
& + \text { permutations }, \quad \text { for } n=-2 .
\end{aligned}
$$

It is important to remark that the squeezed limit of the above expression must be taken with $\ell_{3} \ll \ell_{2} \simeq \ell_{1}$, since this expression has been derived from the wave-number configuration $P \leq Q \leq K$. We see that in this case, the dominant term in the bispectrum (of the order $\log \left(k_{D} \eta_{0} / \ell_{3}\right)$ ) correctly corresponds to the one coming from the dominant term in wave-number space, $P^{2 n+3}$. The permutations must be treated accordingly: for example, for $Q \leq P \leq K$ one has $\ell_{2} \ll \ell_{1} \simeq \ell_{3}$.

For $n \rightarrow-3$, we solve the integrals setting $n=-3$, therefore using approximation (A3) with $m=-1$, and we find then

$$
\begin{aligned}
b_{\ell_{1} \ell_{2} \ell_{3}} & \simeq \frac{\pi^{7} \alpha^{3}}{288} \frac{n(n+3)^{2}}{2 n+3} \frac{\left\langle B^{2}\right\rangle^{3}}{\rho_{\text {rel }}^{3}}\left[\left(\frac{1}{\ell_{1}^{2} \ell_{2}^{2}}+\frac{1}{\ell_{1}^{2} \ell_{3}^{2}}+\frac{1}{\ell_{2}^{2} \ell_{3}^{2}}\right)+\frac{\pi}{16} \frac{n+3}{n+1} k_{D} \eta_{0}\left(\frac{1}{\ell_{1}^{5}}+\frac{1}{2 \ell_{2}^{5}}\right)\right] \\
& + \text { permutations }, \quad \text { for } n \approx-3,
\end{aligned}
$$

where the same considerations as above apply for the squeezed limit. The second term in the above expression, coming from the term proportional to $b(n)$ in Eq. (61) is sub-leading, since it contains a factor $n+3$. Note that since $\left\langle B^{2}\right\rangle \propto(n+3)^{-1}$, the leading term of the bispectrum diverges in $n \rightarrow-3$ as $(n+3)^{-1}$, like the spectrum (cf. Eq. (22) $)$ : this divergence is connected to the infrared divergence of the magnetic energy ${ }^{2}$.

The leading term of the above result Eq. (66), reduced to the squeezed and the equilateral configurations, gives the same result as found in [28] ( $c f$. eqs. (17) and (18) and discussion thereafter, we remind that we use $\alpha=0.1$ ).

\section{ESTIMATION OF THE SIGNAL}

Since the signal-to-noise ratios $(S / N)$ we will be interested in is some function of the maximum multipole a given experiment can reach, $\ell_{\max } \gg 1$, we can use the flat-sky approximation [46, 47] and write for the bispectrum

$$
\left\langle a\left(\vec{\ell}_{1}\right) a\left(\vec{\ell}_{2}\right) a\left(\vec{\ell}_{3}\right)\right\rangle=(2 \pi)^{2} \delta^{(2)}\left(\vec{\ell}_{123}\right) B\left(\ell_{1}, \ell_{2}, \ell_{3}\right)
$$

where $\vec{\ell}_{123}=\vec{\ell}_{1}+\vec{\ell}_{2}+\vec{\ell}_{3}$. With this notation, the reduced bispectrum $b_{\ell_{1} \ell_{2} \ell_{3}}$ coincides with the bispectrum $B\left(\ell_{1}, \ell_{2}, \ell_{3}\right)$.

Our goal now is to quantify the level of NG coming from the stochastic magnetic field and eventually to give a bound on the amplitude of the magnetic field. One way to do it is to exploit the present bound on the primordial local non-Gaussianity parametrized by the quantity $f_{\mathrm{NL}}^{\text {loc }}$. As we mentioned in the introduction, the search for a non-vanishing bispectrum of a local type has given so far a null result and currently $f_{\mathrm{NL}}^{\text {loc }}$ is bounded in the range $-9<f_{\mathrm{NL}}^{\text {loc }}<111$. As the shape of the non-Gaussian signature from the stochastic magnetic field may be different from the one of the local type, one may not directly apply the bounds coming from WMAP5 whose search for nonGaussianity is optimised to search for local primordial contribution. Instead, we proceed in the following way. First, we define the Fisher matrix (see, for example, [42])

$$
F_{i j}=\frac{f_{\text {sky }}}{(2 \pi)^{2} \pi} \int d^{2} \ell_{1} d^{2} \ell_{2} d^{2} \ell_{3} \delta^{(2)}\left(\vec{\ell}_{123}\right) \frac{B_{i}\left(\ell_{1}, \ell_{2}, \ell_{3}\right) B_{j}\left(\ell_{1}, \ell_{2}, \ell_{3}\right)}{6 C\left(\ell_{1}\right) C\left(\ell_{2}\right) C\left(\ell_{3}\right)},
$$

where $f_{\text {sky }}$ is the portion of the observed-sky in a given experiment and $i$ (or $\left.j\right)=(\operatorname{mag}, \operatorname{loc})$. The first entry $F_{\text {mag,mag }}$ of the Fisher matrix corresponds to the signal-to-noise ratio $(S / N)^{2}$ provided by the stochastic magnetic field to the nonGaussianity. We have defined the power spectrum in the flat-sky approximation by $\left\langle a\left(\vec{l}_{1}\right) a\left(\vec{l}_{2}\right)\right\rangle=(2 \pi)^{2} \delta^{(2)}\left(\vec{l}_{12}\right) C\left(\ell_{1}\right)$

\footnotetext{
2 The above expression is valid only for $n \rightarrow-3$ so the denominator is always finite. Note however that the apparent divergence for $n=-3 / 2$ is just an artefact due to our approximation ( $c f$. Eq. (15): $n=-3 / 2$ would correspond to a threshold value for which $\left|\rho_{B}(k)\right|^{2}$ diverges logarithmically for $k \rightarrow 0$ and is not simply white noise.
} 
with $\ell^{2} C(\ell)=\mathcal{A} / \pi$ and $\mathcal{A} \simeq 17.46 \times 10^{-9}$ is the amplitude of the primordial gravitational potential power spectrum computed at first-order. In other words, we assume that the two-point correlation function is dominated by the usual adiabatic contribution from inflation. Finally, the local bispectrum is given by [46]

$$
B_{\mathrm{loc}}\left(\ell_{1}, \ell_{2}, \ell_{3}\right)=\frac{2 f_{\mathrm{NL}}^{\text {loc }} \mathcal{A}^{2}}{\pi^{2}}\left(\frac{1}{\ell_{1}^{2} \ell_{2}^{2}}+\text { cycl. }\right) .
$$

Notice that all these expressions are obtained in the Sachs-Wolfe approximation. We will return back to this point shortly.

Next, we define an effective $f_{\mathrm{NL}}^{\text {eff }}$ which minimises the $\chi^{2}$ defined as

$$
\chi^{2}=\int d^{2} \ell_{1} d^{2} \ell_{2} d^{2} \ell_{3} \delta^{(2)}\left(\vec{\ell}_{123}\right) \frac{\left(\left.f_{\mathrm{NL}}^{\mathrm{eff}} B_{\mathrm{loc}}\left(\ell_{1}, \ell_{2}, \ell_{3}\right)\right|_{f_{\mathrm{NL}}^{\text {loc }}=1}-B_{\mathrm{mag}}\left(\ell_{1}, \ell_{2}, \ell_{3}\right)\right)^{2}}{6 C\left(\ell_{1}\right) C\left(\ell_{2}\right) C\left(\ell_{3}\right)} .
$$

One finds

$$
f_{\mathrm{NL}}^{\mathrm{eff}}=\left.\frac{F_{\mathrm{mag}, \mathrm{loc}}}{F_{\mathrm{loc}, \mathrm{loc}}}\right|_{f_{\mathrm{NL}}^{\mathrm{loc}}=1} .
$$

The signal-to-noise ratio for the primordial local case has already been computed in the flat-sky approximation in Ref. [46]. The result is that $F_{\text {loc,loc }} \simeq\left(4 / \pi^{2}\right) f_{\text {sky }} \mathcal{A}\left(f_{\mathrm{NL}}^{\text {loc }}\right)^{2} \ell_{\max }^{2} \log \left(\ell_{\max } / \ell_{\min }\right)$. The logarithm is typical of scale invariant power spectra and $\ell_{\min }$ is the minimum multipole compatible with the flat-sky approximation. The physical meaning of $f_{\mathrm{NL}}^{\mathrm{eff}}$ is the following: it is the best value of the local $f_{\mathrm{NL}}^{\text {loc }}$ which best mimics the bispectrum from a stochastic magnetic field background. As such, we can apply to this value the current observational limits.

We start with the simplest case $n \approx-3$. Indeed, for $n$ close to -3 , the leading term of the bispectrum is of the same form of the local primordial bispectrum (69) in the squeezed limit $\ell_{3} \ll \ell_{1} \simeq \ell_{2}$ and we immediately find

$$
f_{\mathrm{NL}}^{\mathrm{eff}} \simeq \frac{3 \pi^{9} \alpha^{3}}{288 \mathcal{A}^{2}} \frac{n(n+3)^{2}}{2 n+3} \frac{\left\langle B^{2}\right\rangle^{3}}{\rho_{\mathrm{rel}}^{3}} \simeq 10^{-2}(n+3)^{2}\left(\frac{\left\langle B^{2}\right\rangle}{\left(10^{-9} \text { Gauss }\right)^{2}}\right)^{3}, \quad \text { for } n \approx-3 .
$$

In the case in which the bispectrum is independent from the multipoles, that is for $n>-1$, we find

$$
f_{\mathrm{NL}}^{\mathrm{eff}} \simeq \frac{\pi^{9} \alpha^{3}}{2304 \mathcal{A}^{2}} \frac{(n+3)^{3}}{n+1} \frac{\left\langle B^{2}\right\rangle^{3}}{\rho_{\mathrm{rel}}^{3}}\left(\frac{\ell_{\max }}{\ell_{D}}\right)^{4} \frac{1}{\log \left(\ell_{\max } / \ell_{\min }\right)} \simeq 6 \times 10^{-7} \frac{(n+3)^{3}}{n+1}\left(\frac{\left\langle B^{2}\right\rangle}{\left(10^{-9} \mathrm{Gauss}\right)^{2}}\right)^{3}, \quad \text { for } n>-1 .
$$

Finally, for the case $n=-2$, we find

$$
f_{\mathrm{NL}}^{\mathrm{eff}} \simeq \frac{5 \pi^{10} \alpha^{3}}{2304 \mathcal{A}^{2}} \frac{\left\langle B^{2}\right\rangle^{3}}{\rho_{\mathrm{rel}}^{3}}\left(\frac{\ell_{\mathrm{max}}}{\ell_{D}}\right)^{3} \frac{\log \left(\ell_{D} / \ell_{\max }\right)}{\log \left(\ell_{\max } / \ell_{\min }\right)} \simeq 5 \times 10^{-5}\left(\frac{\left\langle B^{2}\right\rangle}{\left(10^{-9} \mathrm{Gauss}\right)^{2}}\right)^{3}, \quad \text { for } n=-2 .
$$

In all numerical estimates we have taken $\ell_{D}=k_{D} \eta_{0} \simeq 3000, \ell_{\max } \sim 750, \ell_{\min } \sim 10, \alpha \simeq 0.1$, and Eq. (6). We see that the effective value of non-Gaussianity $f_{\mathrm{NL}}^{\text {eff }}$ is smaller than the present upper bound of $\mathcal{O}\left(10^{2}\right)$ on $f_{\mathrm{NL}}^{\text {loc }}$ [2] for magnetic fields $\mathcal{O}(10) \cdot 10^{-9}$ Gauss for $n \approx-3$ and $\mathcal{O}(20) \cdot 10^{-9}$ Gauss for the other cases ${ }^{3}$.

Accounting more precisely for the value of the damping scale $k_{D}$ as a function of the spectral index and of the magnetic field amplitude using Eq. (7), we obtain

$$
\begin{aligned}
& \sqrt{\left\langle B^{2}\right\rangle} \leq 9 \text { nGauss } \text { for } n=-2.9 \\
& \sqrt{\left\langle B^{2}\right\rangle} \leq 25 \text { nGauss } \text { for } n=-2 \\
& \sqrt{\left\langle B^{2}\right\rangle} \leq 20 \text { nGauss } \text { for } n=2
\end{aligned}
$$

3 We have obtained similar estimates repeating the same procedure to define an effective non-Gaussianity parameter starting from a primordial equilateral configuration for which WMAP5 limits exist. In such a case the primordial equilateral configuration is peaked for $\ell_{1} \sim \ell_{2} \sim \ell_{3}$ and the effective non-Gaussianity parameter scales with $\ell_{\max }$ with one power less than the corresponding one obtained from a local primordial bispectrum. 
The corresponding bound on the magnetic field amplitude $B_{\lambda}$ (cf. Eq. (5)) on the scale $\lambda=0.1$ Mpc is unchanged for $n \rightarrow-3$, it becomes $B_{\lambda} \leq 26$ nGauss for $n=-2$, and is less stringent as $n$ grows, becoming irrelevant for $n=2$ : $B_{\lambda} \leq 2 \mu$ Gauss. This is a consequence of the fact that the procedure of using an effective $f_{\mathrm{NL}}$ returns a bound on the integrated magnetic field spectrum, and therefore for very blue spectra the constraint on large scales is irrelevant.

A word of caution is in order here though. In all our estimates, we have used the Sachs-Wolfe approximation for all bispectra. This is certainly a sufficiently good approximation for an experiment like WMAP whose maximum multipole is $\ell_{\max } \sim 750$. This is because the transfer functions for both the scalar contribution to the CMB anisotropies from the stochastic magnetic field and the one from the inflationary adiabatic modes may be taken roughly equal to unity up to $\ell \sim 750$ and they do not affect the computation of the Fisher matrix elements, see [46, 48]. However, for higher multipoles, say $\ell \sim 2000$, typical of an experiment like Planck, the inclusion of the transfer functions will be crucial because the anisotropies from the adiabatic inflationary modes get an exponential suppression due to the Silk damping, while the ones from the scalar modes from the stochastic magnetic field show a much milder suppression [27, 40]. This will increase the value of $f_{\mathrm{NL}}^{\mathrm{eff}}$. Needless to say, the inclusion of the vector and tensor contributions from the magnetic field will help to increase the non-Gaussian signal too.

While writing this paper, the preprint [28] appeared where the computation of the bispectrum from a stochastic magnetic field background was presented for the case $n \approx-3$. Our findings agree with those in Ref. [28] and extend them to other values of the spectral index and by the estimation of the signal-to-noise ratio and of the effective non-Gaussianity parameter.

\section{Acknowledgments}

CC wishes to thank Iain Brown, Ruth Durrer, Martin Kunz, Roy Maartens, Antti Väihkönen and Filippo Vernizzi for helpful discussions. CC acknowledge support from the funding INFN IS PD51 for visiting IASF Bologna.

\section{Appendix A: INTEGRALS OF BESSEL FUNCTIONS}

In order to evaluate both the magnetic field spectrum and bispectrum at large angular scales, we need to evaluate integrals of the type

$$
\int_{0}^{y} d x x^{m} j_{\ell}^{2}(x)
$$

with $y \gg 1$. This integral can be expressed generically in terms of hypergeometric functions; however, good approximations can be found, which are much simpler.

For $m=2$, the integral can be performed exactly: one has

$$
\int_{0}^{y} d x x^{2} j_{\ell}^{2}(x)=\frac{\pi}{4} y^{2}\left[J_{\ell+\frac{1}{2}}^{2}(y)-\frac{2}{y}\left(\ell+\frac{1}{2}\right) J_{\ell+\frac{1}{2}}(y) J_{\ell+\frac{3}{2}}(y)+J_{\ell+\frac{3}{2}}^{2}(y)\right] \simeq \frac{y}{2}
$$

where since $y \gg \ell$ we used the expansion of the Bessel functions for large arguments.

For $m<1$, the integral reaches a constant value for $y \gg \ell$, and can therefore be evaluated in the limit $y \rightarrow \infty$. We find

$$
\begin{aligned}
\int_{0}^{y} d x x^{m} j_{\ell}^{2}(x) \simeq & \frac{1}{4}\left[\frac{\sqrt{\pi} \Gamma\left(\frac{1-m}{2}\right) \Gamma\left(\ell+\frac{m+1}{2}\right)}{\Gamma\left(1-\frac{m}{2}\right) \Gamma\left(\ell+\frac{3-m}{2}\right)}+y^{m-2}\left(\frac{2 y}{m-1}+\sin (\pi \ell-2 y)\right)\right] \stackrel{\ell \gg 1}{\longrightarrow} \frac{\sqrt{\pi} \Gamma\left(\frac{1-m}{2}\right)}{4 \Gamma\left(1-\frac{m}{2}\right)} \ell^{m-1} \\
& \text { for } m<1, y \gg \ell
\end{aligned}
$$

The case $m=1$ is a bit more involved: the integral (A1) grows logarithmically with $y$ and cannot be evaluated with the same approximation as before. In this case we set

$$
\int_{0}^{y} d x x j_{\ell}^{2}(x) \simeq \int_{\ell}^{y} \frac{d x}{x} \cos ^{2}\left(x-\frac{\pi}{2} \ell-\frac{\pi}{4}\right) \simeq \frac{1}{2}[\log (y)-\log (\ell)] \quad \text { for } y \gg \ell .
$$

We are neglecting the subdominant contribution to the integral of the interval $[0, \ell]$, therefore this approximation is slightly underestimating the true result. However, it captures the correct behaviour in $\ell$ and $y$. These approximations are shown in Fig. [4. 

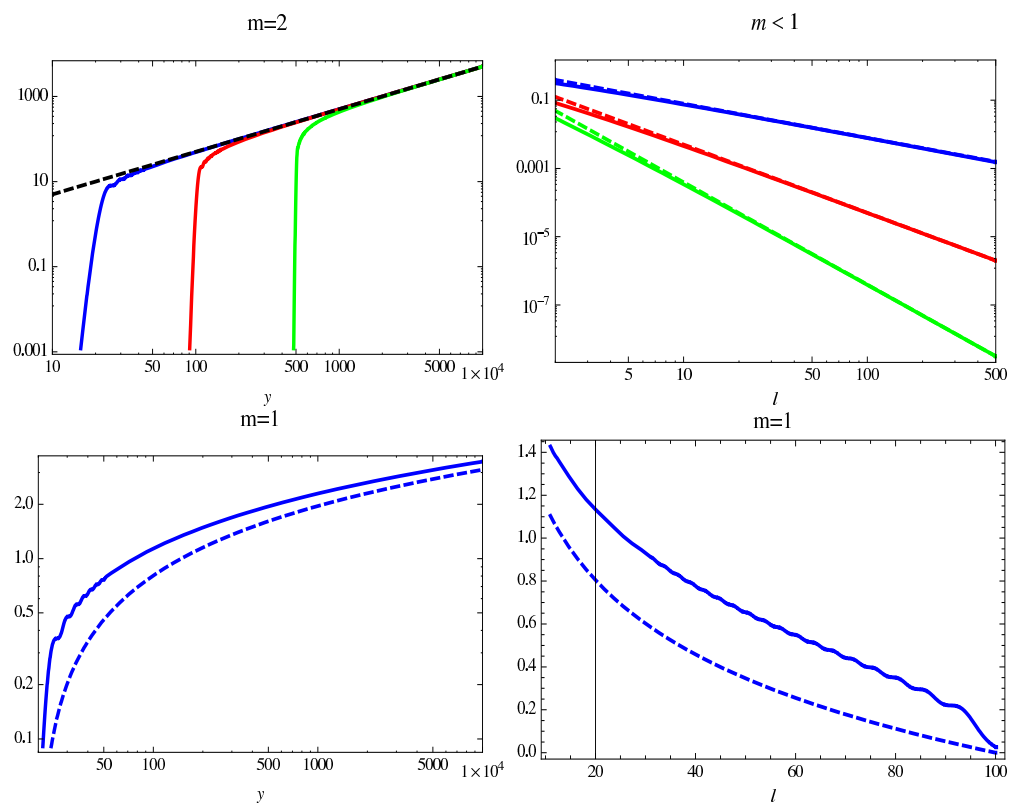

Figure 4: The approximations for the integral in Eq. A1). Upper left plot, for $m=2$ : the integral (solid) and the approximation $y / 2$ (dashed) are shown for $\ell=20, \ell=100, \ell=500$ as a function of $y$. Upper right plot, for $m<1$ : the approximations for $y \gg \ell$ (solid) and for $\ell \gg 1$ (dashed) given in Eq. (A3) are shown as a function of $\ell$ for $m=0, m=-1$ and $m=-2$. Lower plots, for $m=1$ : the integral (solid) and the approximation in Eq. (A4) (dashed) are shown as a function of $y$ for $\ell=20$ (left plot) and as a function of $\ell$ for $y=100$ (right plot).

\section{Appendix B: BISPECTRUM IN COLLINEAR CONFIGURATION}

In the following appendix we describe the technique used to calculate the magnetic energy density bispectrum in the collinear configuration Eq. (42).

Due to the complexity of the calculations we restrict to analytical solutions of the bispectrum integral for two representative spectral indexes: the case $n=2$ (the typical spectrum of a magnetic field generated by a causal mechanism), and the case $n=-2$ (in order to investigate the behaviour of the spectrum also for negative spectral indexes).

The magnetic energy density bispectrum in the collinear configuration is given in Eq. (42). From this we extract the integral in the momenta which is given by the permutation over the three momenta $\mathbf{K}, \mathbf{P}, \mathbf{Q}$ of three basic integrals ${ }^{4}$ :

$$
I(K)=\int d \tilde{K} \int d x\left(I_{a}(K, \tilde{K})+I_{b}(K, \tilde{K})+I_{c}(K, \tilde{K})\right)
$$

\footnotetext{
${ }^{4}$ For simplicity of notation in this appendix we use re-scaled variables: $K=k / k_{D}, Q=q / k_{D}, P=p / k_{D}$ and $\tilde{K}=\tilde{k} / k_{D}$
} 
where $x=\hat{K} \cdot \hat{\tilde{K}}$. The functions $I_{a}(K, \tilde{K}), I_{b}(K, \tilde{K}), I_{c}(K, \tilde{K})$ are:

$$
\begin{aligned}
I_{a}(K, \tilde{K})= & \tilde{K}^{2+n}\left(\frac{K^{2}}{4}+\tilde{K}^{2}+K \tilde{K} x\right)^{\frac{n}{2}}\left(K^{2}+\tilde{K}^{2}+2 K \tilde{K} x\right)^{-1+\frac{n}{2}} \\
& \left(\frac{8 \tilde{K}^{4}+24 K \tilde{K}^{3} x+K^{4}\left(1+x^{2}\right)+3 K^{3} \tilde{K} x\left(3+x^{2}\right)+K^{2} \tilde{K}^{2}\left(7+19 x^{2}\right)}{K^{2}+4 \tilde{K}^{2}+4 K \tilde{K} x}\right) \\
I_{b}(K, \tilde{K})= & \tilde{K}^{2+n}\left(\frac{K^{2}}{4}+\tilde{K}^{2}+K \tilde{K} x\right)^{n / 2}\left(\tilde{K}^{2}+\frac{1}{4} K(K-4 \tilde{K} x)\right)^{n / 2} \\
& \left(\frac{\left(32 \tilde{K}^{4}+4 K^{2} \tilde{K}^{2}\left(1-5 x^{2}\right)+K^{4}\left(1+x^{2}\right)\right)}{\left(\left(K^{2}+4 \tilde{K}^{2}\right)^{2}-16 K^{2} \tilde{K}^{2} x^{2}\right)}\right) \\
I_{c}(K, \tilde{K})= & \tilde{K}^{2+n}\left(K^{2}+\tilde{K}^{2}-2 K \tilde{K} x\right)^{-1+\frac{n}{2}}\left(\tilde{K}^{2}+\frac{1}{4} K(K-4 \tilde{K} x)\right)^{n / 2} \\
& \left(\frac{\left(8 \tilde{K}^{4}-24 K \tilde{K}^{3} x+K^{4}\left(1+x^{2}\right)-3 K^{3} \tilde{K} x\left(3+x^{2}\right)+K^{2} \tilde{K}^{2}\left(7+19 x^{2}\right)\right)}{\left(K^{2}+4 \tilde{K}^{2}-4 K \tilde{K} x\right)}\right)
\end{aligned}
$$

We note that due to the symmetry $\tilde{K} \rightarrow-\tilde{K}$ we have that the first and the third integrals are indeed the same $I_{a}(K)=I_{c}(K)$, therefore to obtain the energy density bispectrum in the collinear configuration we need to solve only the two integrals of $I_{a}(K, \tilde{K})$ and $I_{b}(K, \tilde{K})$.

\section{Integration Domains}

The sharp cut-off of the PMF spectrum at the damping scale $k_{D}$, imposed to account for the magnetic fields suppression on small scales, leads to many conditions on the angle $\hat{\tilde{K}} \cdot \hat{K}$. This causes the integration domain to be split into various sub-domains. The conditions are different for $I_{a}$ and $I_{b}$, therefore for simplicity in the following we consider the two integrations separately.

\section{Domains of $I_{a}$}

The sharp cut off imposes:

$$
\begin{aligned}
\tilde{K} & <1 \\
\left(\frac{K^{2}}{4}+\tilde{K}^{2}+K \tilde{K} x\right) & <1 \\
\left(K^{2}+\tilde{K}^{2}+2 K \tilde{K} x\right) & <1
\end{aligned}
$$

This leads to the following integration scheme:

$$
\begin{aligned}
& \text { 1) } 0<K<1 \\
& \int_{0}^{1-K} d \tilde{K} \int_{-1}^{1} d x I_{a}(\tilde{K}, K)+\int_{1-K}^{1} d \tilde{K} \int_{-1}^{\frac{1-K^{2}-\tilde{K}^{2}}{2 K \tilde{K}}} d x I_{a}(\tilde{K}, K) \\
& \text { 2) } 1<K<2 \\
& \int_{K-1}^{1} d \tilde{K} \int_{-1}^{\frac{1-K^{2}-\tilde{K}^{2}}{2 K \tilde{K}}} d x I_{a}(\tilde{K}, K)
\end{aligned}
$$




\section{Domains of $I_{b}$}

The sharp cut off imposes:

$$
\begin{aligned}
\tilde{K} & <1 \\
\left(\frac{K^{2}}{4}+\tilde{K}^{2}+K \tilde{K} x\right) & <1 \\
\left(\frac{K^{2}}{4}+\tilde{K}^{2}-K \tilde{K} x\right) & <1
\end{aligned}
$$

This leads to the following integration scheme for $0<K<2$ :

$$
\int_{0}^{\frac{2-K}{2}} d \tilde{K} \int_{-1}^{1} d x I_{b}(\tilde{K}, K)+\int_{\frac{2-K}{2}}^{\frac{\sqrt{4-K^{2}}}{2}} d \tilde{K} \int_{\frac{-1+K^{2} / 4+\tilde{K}^{2}}{K K}}^{\frac{1-K^{2} / 4-\tilde{K}^{2}}{K \tilde{K}}} d x I_{b}(\tilde{K}, K)
$$

in the interval $\frac{\sqrt{4-K^{2}}}{2}<\tilde{K}<1$ the integral collapses to zero.

\section{4. $\mathrm{n}=\mathbf{2}$}

First we consider the case $n=2$ which is the easiest from the point of view of the calculations. In fact the angular integrand functions for this spectral index simply reduce to:

$$
\begin{aligned}
& I_{a}(K, \tilde{K}, x)=\frac{1}{4} \tilde{K}^{4}\left(8 \tilde{K}^{4}+24 K \tilde{K}^{3} x+K^{4}\left(1+x^{2}\right)+3 K^{3} \tilde{K} x\left(3+x^{2}\right)+K^{2} \tilde{K}^{2}\left(7+19 x^{2}\right)\right) \\
& I_{b}(K, \tilde{K}, x)=\frac{1}{16} \tilde{K}^{4}\left(32 \tilde{K}^{4}+4 K^{2} \tilde{K}^{2}\left(1-5 x^{2}\right)+K^{4}\left(1+x^{2}\right)\right)
\end{aligned}
$$

Once performed the angular integrations, following the integration scheme reported in the previous paragraph, the radial integrations become trivial and the result is:

$$
\left.I(K)\right|_{n=2}=\left(\frac{4}{3}-3 K+\frac{20 K^{2}}{7}-\frac{23 K^{3}}{16}+\frac{2 K^{4}}{5}-\frac{K^{5}}{16}+\frac{K^{7}}{256}-\frac{17 K^{9}}{53760}\right)
$$

In Fig. 2 we have shown the result for $n=2$. We note that, as it happens for the energy density spectrum, also the PMF energy density bispectrum goes to zero for $K=2$ as expected.

\section{5. $\mathrm{n}=-2$}

Here we consider the case $n=-2$. The functions $I_{a}$ and $I_{b}$ for this spectral index reduce to:

$$
\begin{aligned}
I_{a}(K, \tilde{K}, x) & =\frac{4\left(8 \tilde{K}^{4}+24 K \tilde{K}^{3} x+K^{4}\left(1+x^{2}\right)+3 K^{3} \tilde{K} x\left(3+x^{2}\right)+K^{2} \tilde{K}^{2}\left(7+19 x^{2}\right)\right)}{\left(K^{2}+\tilde{K}^{2}+2 K \tilde{K} x\right)^{2}\left(K^{2}+4 \tilde{K}^{2}+4 K \tilde{K} x\right)^{2}} \\
I_{b}(K, \tilde{K}, x) & =\frac{16\left(32 \tilde{K}^{4}+4 K^{2} \tilde{K}^{2}\left(1-5 x^{2}\right)+K^{4}\left(1+x^{2}\right)\right)}{\left(\left(K^{2}+4 \tilde{K}^{2}\right)^{2}-16 K^{2} \tilde{K}^{2} x^{2}\right)^{2}}
\end{aligned}
$$

We note how these functions are far more complicated than the ones for the $n=2$ case. Once performed the angular integrations in both the integrals we have the appearance of absolute values like $|K-2 \tilde{K}|$ and $|K-\tilde{K}|$, their presence influences the integration domains creating further splitting into several sub-domains. Since we are interested in the effect on CMB where only the low $K$ part of the spectrum has a role we restrict ourselves to the $K<1 / 2$ region of the spectrum. The analytical result for $n=-2$ unfortunately has a very long and complicated form, therefore, for the sake of simplicity, we show only the infrared limit:

$$
\begin{aligned}
& I_{a}(K) \sim \frac{24.674}{K^{3}} \\
& I_{b}(K) \sim \frac{24.674}{K^{3}} \\
& I(K) \sim \frac{73.8367}{K^{3}}
\end{aligned}
$$


Fig. 2 shows the exact result.

[1] For a review, see D. H. Lyth and A. Riotto, Phys. Rept. 314, 1 (1999).

[2] E. Komatsu et al. [WMAP Collaboration], arXiv:0803.0547] [astro-ph].

[3] See http://planck.esa.int/.

[4] N. Bartolo, E. Komatsu, S. Matarrese and A. Riotto, Phys. Rept. 402, 103 (2004).

[5] V. Acquaviva, N. Bartolo, S. Matarrese and A. Riotto, Nucl. Phys. B 667, 119 (2003); J. Maldacena, JHEP 0305, 013 (2003).

[6] N. Bartolo, S. Matarrese and A. Riotto, Phys. Rev. D 65, 103505 (2002); F. Bernardeau and J. P. Uzan, Phys. Rev. D 66, 103506 (2002); F. Vernizzi and D. Wands, JCAP 0605, 019 (2006).

[7] D. H. Lyth, C. Ungarelli and D. Wands, Phys. Rev. D 67 (2003) 023503.

[8] N. Bartolo, S. Matarrese and A. Riotto, Phys. Rev. D 69, 043503 (2004).

[9] T. Hamazaki and H. Kodama, Prog. Theor. Phys. 96 (1996) 1123.

[10] G. Dvali, A. Gruzinov and M. Zaldarriaga, Phys. Rev. D 69, 023505 (2004); L. Kofman, arXiv:astro-ph/0303614

[11] G. Dvali, A. Gruzinov and M. Zaldarriaga, Phys. Rev. D 69 (2004) 083505.

[12] D. H. Lyth, JCAP 0511 (2005) 006; M. P. Salem, Phys. Rev. D 72 (2005) 123516; D. H. Lyth and A. Riotto, Phys. Rev. Lett. 97 (2006) 121301.

[13] M. Bastero-Gil, V. Di Clemente and S. F. King, Phys. Rev. D 70, 023501 (2004); E. W. Kolb, A. Riotto and A. Vallinotto, Phys. Rev. D 71, 043513 (2005); E. W. Kolb, A. Riotto and A. Vallinotto, Phys. Rev. D 73, 023522 (2006).

[14] M. Alishahiha, E. Silverstein and D. Tong, Phys. Rev. D 70, 123505 (2004) arXiv:hep-th/0404084.

[15] N. Arkani-Hamed, P. Creminelli, S. Mukohyama and M. Zaldarriaga, JCAP 0404, 001 (2004) arXiv:hep-th/0312100.

[16] D. Babich, P. Creminelli and M. Zaldarriaga, JCAP 0408, 009 (2004) arXiv:astro-ph/0405356.

[17] P. Creminelli, A. Nicolis, L. Senatore, M. Tegmark and M. Zaldarriaga, JCAP 0605, 004 (2006) arXiv:astro-ph/0509029.

[18] A. Curto, E. Martinez-Gonzalez, P. Mukherjee, R. B. Barreiro, F. K. Hansen, M. Liguori and S. Matarrese, arXiv:0807.0231 [astro-ph].

[19] D. Grasso and H. R. Rubinstein, Phys. Rept. 348 (2001) 163 arXiv:astro-ph/0009061; M. Giovannini, Int. J. Mod. Phys. D 13 (2004) 391 arXiv:astro-ph/0312614

[20] A. Kosowsky, T. Kahniashvili, G. Lavrelashvili and B. Ratra, Phys. Rev. D 71 (2005) 043006

[21] C. Caprini, R. Durrer and T. Kahniashvili, Phys. Rev. D 69 (2004) 063006 arXiv:astro-ph/0304556.

[22] G. Chen, P. Mukherjee, T. Kahniashvili, B. Ratra and Y. Wang, Astrophys. J. 611 (2004) 655 arXiv:astro-ph/0403695; P. D. Naselsky, L. Y. Chiang, P. Olesen and O. V. Verkhodanov, Astrophys. J. 615 (2004) 45 arXiv:astro-ph/0405181; P. Naselsky and J. Kim, arXiv:0804.3467 [astro-ph].

[23] R. Durrer, T. Kahniashvili and A. Yates, Phys. Rev. D 58 (1998) 123004 arXiv:astro-ph/9807089; see also T. Kahniashvili, G. Lavrelashvili and B. Ratra, Phys. Rev. D 78 (2008) 063012 arXiv:0807.4239 [astro-ph]].

[24] M. Demianski and A. G. Doroshkevich, Phys. Rev. D 75 (2007) 123517 arXiv:astro-ph/0702381; A. Bernui and W. S. Hipolito-Ricaldi, arXiv:0807.1076 [astro-ph]; A. Bernui, Phys. Rev. D 78 (2008) 063531 arXiv:0809.0934 [astro$\mathrm{ph}]$.

[25] I. Brown and R. Crittenden, Phys. Rev. D 72 (2005) 063002 arXiv:astro-ph/0506570; I. A. Brown, arXiv:0812.1781 [astro-ph].

[26] R. Holman and A. J. Tolley, JCAP 0805 (2008) 001 arXiv:0710.1302 [hep-th]].

[27] F. Finelli, F. Paci and D. Paoletti, Phys. Rev. D 78 (2008) 023510 arXiv:0803.1246 [astro-ph].

[28] T. R. Seshadri and K. Subramanian, arXiv:0902.4066 [astro-ph.CO].

[29] T. Kahniashvili and B. Ratra, Phys. Rev. D 75 (2007) 023002 arXiv:astro-ph/0611247.

[30] W. Hu and M. J. White, Phys. Rev. D 56 (1997) 596 arXiv:astro-ph/9702170.

[31] K. Jedamzik, V. Katalinic and A. V. Olinto, Phys. Rev. D 57 (1998) 3264 arXiv:astro-ph/9606080.

[32] K. Subramanian and J. D. Barrow, Phys. Rev. D 58 (1998) 083502 arXiv:astro-ph/9712083.

[33] R. Durrer, P. G. Ferreira and T. Kahniashvili, Phys. Rev. D 61 (2000) 043001 arXiv:astro-ph/9911040].

[34] R. Durrer and C. Caprini, JCAP 0311 (2003) 010 arXiv:astro-ph/0305059.

[35] C. J. Hogan, Phys. Rev. Lett. 51, (1983) 1488; J. M. Quashnock et al, Astrophys. J. 344 (1989) L49; B. Cheng and A.V. Olinto, Phys. Rev. D 50 (1994) 2412; G. Baym et al., Phys. Rev. D 53 (1996) 662; G. Sigl et al., Phys. Rev. D 55 (1997) 4582; D. Boyanovsky and H. J. de Vega, AIP Conf. Proc. 784 (2005) 434 arXiv:astro-ph/0502212; T. Vachaspati, Phys. Lett. B 265 (1991) 258; K. Enqvist and P. Olesen, Phys. Lett. B 319 (1993) 178 arXiv:hep-ph/9308270; M. Hindmarsh and A. Everett, Phys. Rev. D 58 (1998) 103505 arXiv:astro-ph/9708004]; D. Grasso and A. Riotto, Phys. Lett. B 418 (1998) 258 arXiv:hep-ph/9707265; J. M. Cornwall, Phys. Rev. D 56 (1997) 6146; T. Vachaspati, Phys. Rev. Lett. 87 (2001) 251302; M. Joyce and M. E. Shaposhnikov, Phys. Rev. Lett. 79 (1997) 1193 arXiv:astro-ph/9703005; G. B. Field and S. M. Carroll, Phys. Rev. D 62 (2000) 103008 arXiv:astro-ph/9811206; L. Campanelli and M. Giannotti, Phys. Rev. D 72 (2005) 123001 arXiv:astro-ph/0508653.

[36] E. R. Harrison, Mon. Not. Roy. Astron. Soc. 147 (1970) 279; E. R. Harrison, Phys. Rev. Lett. 30 (1973) 188; S. Matarrese, S. Mollerach, A. Notari and A. Riotto, Phys. Rev. D 71 (2005) 043502 arXiv:astro-ph/0410687; R. Gopal and S. Sethi, Mon. Not. Roy. Astron. Soc. 363 (2005) 529 arXiv:astro-ph/0411170; K. Takahashi, K. Ichiki, H. Ohno and H. Hanayama, 
Phys. Rev. Lett. 95 (2005) 121301 arXiv:astro-ph/0502283]; T. Kobayashi, R. Maartens, T. Shiromizu and K. Takahashi, Phys. Rev. D 75 (2007) 103501 arXiv:astro-ph/0701596.

[37] M. S. Turner and L. M. Widrow, Phys. Rev. D 37, (1988) 2743; B. Ratra, Astrophys. J. Lett. 391 (1992) L1; A. Davis et al., Phys. Lett. B 501, (2001) 165; F. Finelli and A. Gruppuso, Phys. Lett. B 502, (2001) 216;

T. Prokopec, O. Tornqvist and R. Woodard, Phys. Rev. Lett. 89 (2002) 101301; J. Martin and J. Yokoyama, arXiv:0711.4307 [astro-ph].

[38] M. Gasperini, M. Giovannini and G. Veneziano, Phys. Rev. Lett. 75 (1995) 3796 arXiv:hep-th/9504083; D. Lemoine and M. Lemoine, Phys. Rev. D 52 (1995) 1955; K. Bamba and J. Yokoyama, Phys. Rev. D 69 (2004) 043507 arXiv:astro-ph/0310824;

[39] C. Caprini and R. Durrer, Phys. Rev. D 65 (2001) 023517 arXiv:astro-ph/0106244.

[40] D. Paoletti, F. Finelli and F. Paci, arXiv:0811.0230 [astro-ph], to be published in MNRAS

[41] R. Durrer, The Cosmic Microwave Background, Cambridge University Press 2008

[42] E. Komatsu and D. N. Spergel, Phys. Rev. D 63 (2001) 063002 arXiv:astro-ph/0005036.

[43] L. M. Wang and M. Kamionkowski, Phys. Rev. D 61 (2000) 063504 arXiv:astro-ph/9907431.

[44] J. R. Fergusson and E. P. S. Shellard, Phys. Rev. D 76 (2007) 083523 arXiv:astro-ph/0612713.

[45] I.S. Gradshteyn and I.M. Ryzhik, Table of Integrals, Series, and Products, Academic Press, New York and London (1965).

[46] D. Babich and M. Zaldarriaga, Phys. Rev. D 70, 083005 (2004) arXiv:astro-ph/0408455.

[47] W. Hu, Phys. Rev. D 62, 043007 (2000) arXiv:astro-ph/0001303.

[48] N. Bartolo and A. Riotto, arXiv:0811.4584 [astro-ph]. 